\title{
Power Allocation and Parameter Estimation for Multipath-based 5G Positioning
}

\author{
Anastasios Kakkavas, Student Member, IEEE, \\ Henk Wymeersch, Senior Member, IEEE, \\ Gonzalo Seco-Granados, Senior Member, IEEE, \\ Mario H. Castañeda García, Member, IEEE, \\ Richard A. Stirling-Gallacher, Member, IEEE, \\ and Josef A. Nossek, Life Fellow, IEEE
}

\begin{abstract}
We consider a single-anchor multiple-input multiple-output orthogonal frequency-division multiplexing system with imperfectly synchronized transmitter (Tx) and receiver (Rx) clocks, where the Rx estimates its position based on the received reference signals. The Tx, having (imperfect) prior knowledge about the Rx location and the surrounding geometry, transmits the reference signals based on a set of fixed beams. In this work, we develop strategies for the power allocation among the beams
\end{abstract}

This work was supported in part by the EU-H2020 project Fifth Generation Communication Automotive Research and Innovation (5GCAR), and in part by the ICREA Academia program and the Spanish Ministry of Science, Innovation and Universities project TEC2017-89925-R.

A. Kakkavas is with the Munich Research Center, Huawei Technologies Duesseldorf GmbH, 80992 Munich, Germany, and also with the Department of Electrical and Computer Engineering, Technical University of Munich, 80333 Munich, Germany (e-mail: anastasios.kakkavas@huawei.com).

H. Wymeersch is with the Department of Electrical Engineering, Chalmers University of Technology, 41258 Gothenburg, Sweden (email: henkw@chalmers.se).

G. Seco-Granados is with the Department of Telecommunications and Systems Engineering, Universitat Autonoma de Barcelona, Spain (UAB) (e-mail: gonzalo.seco@uab.cat).

M. H. Castañeda García and R. A. Stirling-Gallacher are with the Munich Research Center, Huawei Technologies Duesseldorf GmbH, 80992 Munich, Germany (e-mail: mario.castaneda@huawei.com; richard.sg@ huawei.com).

J. A. Nossek is with the Department of Electrical and Computer Engineering, Technical University of Munich, 80333 Munich, Germany (e-mail: josef.a.nossek@tum.de).

This work has been submitted to the IEEE for possible publication. Copyright may be transferred without notice, after which this version may no longer be accessible. 
aiming to minimize the expected Cramér-Rao lower bound for Rx positioning. Additional constraints on the design are included to ensure that the line-of-sight (LOS) path is detected with high probability. Furthermore, the effect of clock asynchronism on the resulting allocation strategies is also studied. We also propose a gridless compressed sensing-based position estimation algorithm, which exploits the information on the clock offset provided by non-line-of-sight paths, and show that it is asymptotically efficient.

\section{Index Terms}

positioning, localization, 5G, reference signal, power allocation, parameter estimation

\section{INTRODUCTION}

With the advent of fifth generation (5G) mobile networks, positioning has attracted lots of research interest. The large chunks of bandwidth available at millimeter-wave (mm-Wave) frequencies, as well as the potentially large number of antennas placed at both sides of the communication link are the main driving forces, not only for very high data rates and massive connectivity [1], [2], but also for a drastic improvement of the positioning accuracy of cellular networks [3]. Recently, within the Third Generation Partnership Project (3GPP), besides positioning techniques already existing in previous generations of cellular networks [4], such as observed time difference of arrival (OTDOA), uplink TDOA (UTDOA), new techniques have been standardized, including downlink (DL)-angle of departure (AOD), uplink (UL)-angle of arrival (AOA) and multi-cell round-trip time (RTT) [5]. In addition, proposals for reporting delay and angular multipath measurements to enable single-anchor positioning have been considered [6]. With their enhanced positioning capabilities, 5G systems aim to accommodate use cases like assisted/autonomous driving [7], augmented reality and industrial internet of things (IoT) [6].

Single-anchor localization, that leverages the high temporal and angular resolution of mmWave multiple-input multiple-output (MIMO) systems, has received increasing attention in recent years, as it has the potential to ease the requirements of multi-anchor hearability and interference management. The fundamental limits of single-anchor positioning have been investigated in [8] for line-of-sight (LOS) and [9]-[12] for multipath channels with single-bounce-non-LOS (NLOS) components.

The single-anchor localization algorithms in the literature can be classified into two categories: one-shot schemes without tracking [13]-[20], and tracking approaches [21] $-[27]$. While the latter 
mainly focus on positon estimation and tracking given the channel parameter measurements, the former also deal with the estimation of the channel parameters, as done in the present work. A three-stage algorithm for position estimation with a multiple-input multiple-output (MIMO)orthogonal frequency-division multiplexing (OFDM) system was proposed in [13], where in the first stage a compressed sensing-based algorithm is used to obtain coarse estimates of the multipath parameters (number of paths, times of arrival (TOAs), AODs, AOAs and gains), with the coarse estimates refined in the second stage. In the third stage, the refined estimates are mapped to the receiver $(\mathrm{Rx})$ position and orientation and the scatterer/reflector positions using the extended invariance principle (EXIP). A similar approach is followed in [14], with the main difference lying in the mapping from channel parameters to position parameters, where an iterative Gibbs sampling method is employed. In [15] range-free angle-based approaches are developed assuming prior map information. An algorithm for localization and synchronization of cooperating full-duplex agents using a single-anchor is developed in [16]. The authors of [17] propose a protocol and an accompanying algorithm that enables a single-anchor to (quasi)simultaneously receive messages from multiple agents in order to localize them using TOA and AOA measurements. The proposed approach is verified on an experimental setup. A DL positioning algorithm for a single-antenna Rx, based on TOA and AOD measurements is proposed in [19]. The work is extended in [20], where a two-step process is used, with the coarse parameter estimates obtained in the first step used for adaptation of the transmitter (Tx) beamforming matrix in the second step. In [28] an iterative position estimation and Tx beamforming refinement algorithm is developed.

Similar to [20], [28], many works have considered the use of prior knowledge of the $\mathrm{Rx}$ position at the Tx to design beamformers that improve the Rx's localization accuracy. In [29] Cramér-Rao lower bound (CRLB)-optimal precoders for tracking the AOD and AOA of a path were designed, taking the uncertainty about their value into account. In [30], assuming a LOS channel and a multicarrier system, beamformers minimizing the TOA and AOA error bounds were proposed, based on the current estimate of the Rx position. Using a similar setup, but additionally considering multiple users, the authors of [31] designed beamformers maximizing a weighted sum of Fisher information on delay, AOD and AOA. Although in a different context, the algorithms and the conclusions of [32], [33] are relevant to our Tx beamforming problem. In [32], [33], robust beamformers under angular uncertainty were designed and it is concluded that the Rx steering vector and its derivative contain all the localization information. Again in a 
different but still relevant setup, the authors of [34] and [35] compute the optimal power allocation among multiple anchors for ranging-based localization by solving a semidefinite program (SDP). In [36] it was shown that, when the uncertainty about the Rx position is not considered, it is optimal to transmit only on the directions corresponding to the Tx array steering vector and its derivative. The power allocation among these two directions minimizing the squared position error bound (SPEB) qas analytically calculated in [36]. When the Rx location uncertainty is taken into account, the optimal power allocation among the beams of a given Tx beam codebook was computed to minimize the average or maximum SPEB.

In this paper, we extend our work in [36]. We consider a single-anchor setup and a sparse multipath channel, which comprises the LOS path and a number of single-bounce NLOS paths, as multi-bounce paths are considered too weak for reception at mm-Wave frequencies [37]-[40]. The Tx has only a coarse prior knowledge of the underlying geometry and in addition, the TxRx clocks are imperfectly synchronized. We optimize the power allocation on a beam codebook for the multipath channel and examine the effect of imperfect synchronization on the resulting power allocation. Also, we develop a novel position estimation algorithm, which is evaluated for the proposed power allocation strategies. The main contributions of the work can be summarized as follows:

- We propose power allocation strategies on a fixed Tx beam codebook with the aim of minimizing the expected positioning error of the Rx. The optimal solution and a suboptimal one with lower computational complexity are presented and evaluated.

- We develop a two-stage position estimation algorithm. The first stage consists of a gridless channel parameter estimation algorithm, based on [41]. The second stage maps the channel parameter estimates to position parameters. The information about the clock offset offered by NLOS paths in combination with the LOS path is exploited so as to discard false alarms.

The rest of the paper is organized as follows. In Sec. II we present the system model and the assumptions of the work. The theoretical bound on positioning accuracy is briefly discussed in Sec. III and the proposed power allocation methods are presented in Sec. IV] The position estimation algorithm is introduced in Sec. $\mathrm{V}$ and numerical evaluations of the proposed approaches are provided in Sec. VI. Sec. VII concludes the work.

Notation: We use bold lowercase for vectors, bold uppercase for matrices, non-bold for scalars and calligraphic letters for sets. Depending on its argument, $|\cdot|$ denotes the absolute value of a scalar, the determinant of a matrix or the cardinality of a set. The transpose, conjugate transpose 


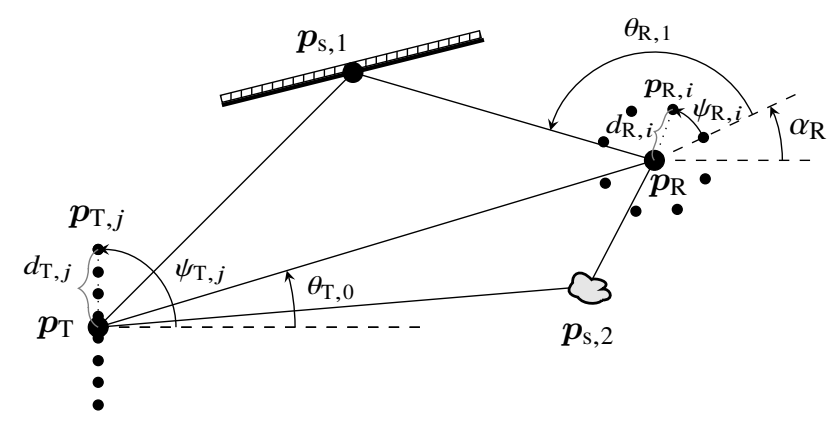

Fig. 1. Geometric model, example with a uniform linear array (ULA) at the Tx and a uniform circular array (UCA) at the Rx.

and $p$-norm of a vector/matrix are denoted by $(\cdot)^{\mathrm{T}},(\cdot)^{\mathrm{H}}$ and $\|\cdot\|_{p}$ and the Frobenius norm of a matrix is denoted by $\|\cdot\|_{F} \cdot \mathfrak{R}\{\cdot\}$ and $\mathfrak{J}\{\cdot\}$ denote the real and imaginary part of a complex number and $\arg (\cdot)$ denotes its phase. The $i$-th element of a vector and the $(i, j)$-th element of a matrix are denoted by $[\cdot]_{i}$ and $[\cdot]_{i, j}$, respectively. $\boldsymbol{I}_{n}, \mathbf{1}$ and $\mathbf{0}$ denote the identity matrix of size $n$, and the all-ones all-zeros matrix of the appropriate size $\operatorname{diag}(\boldsymbol{x})$ denotes the diagonal matrix with the elements of $\boldsymbol{x}$ on its diagonal. The expectation operator is denoted by $\mathbb{E}[\cdot]$ and the sets of real and complex numbers are denoted by $\mathbb{R}$ and $\mathbb{C}$. A multivariate (circularly symmetric complex) Gaussian distribution with mean $\boldsymbol{\mu}$ and covariance matrix $C$ is denoted by $\mathcal{N}(\boldsymbol{\mu}, \boldsymbol{C})$ $\left(\mathcal{N}_{\mathbb{C}}(\boldsymbol{\mu}, \boldsymbol{C})\right)$. The Hessian of a function $f(\boldsymbol{x})$ is denoted as $D_{\boldsymbol{x}}^{2} f(\boldsymbol{x})$.

\section{SyStem Model AND Assumptions}

\section{A. Geometric Model}

The Tx consists of an array with $N_{\mathrm{T}}$ antennas and reference point located at the origin. The Rx consists of an array with $N_{\mathrm{R}}$ antennas, a reference point located at $\boldsymbol{p}_{\mathrm{R}}=\left[p_{\mathrm{R}, \mathrm{x}}, p_{\mathrm{R}, \mathrm{y}}\right]^{\mathrm{T}} \in \mathbb{R}^{2}$ and orientation $\alpha_{\mathrm{R}}$. The position of the $j$-th element of the Tx array is given by

$$
\boldsymbol{p}_{\mathrm{T}, j}=d_{\mathrm{T}, j} \boldsymbol{u}\left(\psi_{\mathrm{T}, j}\right) \in \mathbb{R}^{2}, \quad j=0, \ldots, N_{\mathrm{T}}-1,
$$

where $\boldsymbol{u}(\psi)=[\cos (\psi), \sin (\psi)]^{\mathrm{T}}$ and $d_{\mathrm{T}, j}$ and $\psi_{\mathrm{T}, j}$ are its distance and angle from the Tx array's reference point, as shown in Fig. 1. Accordingly, the position of the $i$-th element of the Rx array is given by

$$
\boldsymbol{p}_{\mathrm{R}, i}=d_{\mathrm{R}, i} \boldsymbol{u}\left(\psi_{\mathrm{R}, i}+\alpha_{\mathrm{R}}\right) \in \mathbb{R}^{2}, \quad i=0, \ldots, N_{\mathrm{R}}-1 .
$$

We assume that for all antenna pairs there are $L$ discrete propagation paths. The first of these $L$ paths $(l=0)$ is the LOS path and the rest $(l=1, \ldots, L-1)$ are single-bounce NLOS paths. 
The point of incidence of the $l$-th single-bounce path, which corresponds either to scattering or reflection, is $\boldsymbol{p}_{\mathrm{s}, l}=\left[p_{\mathrm{s}, l, \mathrm{x}}, p_{\mathrm{s}, l, \mathrm{y}}\right]^{\mathrm{T}}, l=1, \ldots, L-1$. The array apertures are assumed to be small compared to the distance between Tx and Rx, as well as the distance between each of the scatterers/reflectors and the Tx or Rx. Therefore, the delay of the $l$-th path from Tx element $j$ to Rx element $i$ can be approximated by [12]

$$
\tau_{l, i, j} \approx \tau_{l}-\tau_{\mathrm{T}, j}\left(\theta_{\mathrm{T}, l}\right)-\tau_{\mathrm{R}, i}\left(\theta_{\mathrm{R}, l}\right), \quad l=0, \ldots, L-1,
$$

where

$$
\begin{aligned}
\tau_{l} & = \begin{cases}\left\|\boldsymbol{p}_{\mathrm{R}}\right\|_{2} / c+\epsilon_{\mathrm{clk}}, & l=0 \\
\left(\left\|\boldsymbol{p}_{\mathrm{s}, l}\right\|_{2}+\left\|\boldsymbol{p}_{\mathrm{R}}-\boldsymbol{p}_{\mathrm{s}, l}\right\|_{2}\right) / c+\epsilon_{\mathrm{clk}}, & l \neq 0,\end{cases} \\
\tau_{\mathrm{T}, j}\left(\theta_{\mathrm{T}, l}\right) & =d_{\mathrm{T}, j} \boldsymbol{u}^{\mathrm{T}}\left(\psi_{\mathrm{T}, j}\right) \boldsymbol{u}\left(\theta_{\mathrm{T}, l}\right) / c, \\
\tau_{\mathrm{R}, i}\left(\theta_{\mathrm{R}, l}\right) & =d_{\mathrm{R}, i} \boldsymbol{u}^{\mathrm{T}}\left(\psi_{\mathrm{R}, i}\right) \boldsymbol{u}\left(\theta_{\mathrm{R}, l}\right) / c,
\end{aligned}
$$

with $\epsilon_{\mathrm{clk}}$ being the clock offset between $\mathrm{Tx}$ and $\mathrm{Rx}$ and $c$ the speed of light. The angles are defined as

$$
\begin{aligned}
& \theta_{\mathrm{T}, l}= \begin{cases}\operatorname{atan} 2\left(p_{\mathrm{R}, \mathrm{y}}, p_{\mathrm{R}, \mathrm{x}}\right), & l=0 \\
\operatorname{atan} 2\left(p_{\mathrm{s}, l, \mathrm{y}}, p_{\mathrm{s}, l, \mathrm{x}}\right), & l \neq 0\end{cases} \\
& \theta_{\mathrm{R}, l}= \begin{cases}\theta_{\mathrm{T}, l}+\pi-\alpha_{\mathrm{R}}, & l=0, \\
\operatorname{atan} 2\left(p_{\mathrm{s}, l, \mathrm{y}}-p_{\mathrm{R}, \mathrm{y}}, p_{\mathrm{s}, l, \mathrm{x}}-p_{\mathrm{R}, \mathrm{x}}\right)-\alpha_{\mathrm{R}}, & l \neq 0,\end{cases}
\end{aligned}
$$

with $\operatorname{atan} 2(y, x)$ being the four-quadrant inverse tangent function.

\section{B. Signal Model}

An OFDM waveform with subcarrier spacing $\Delta f, N$ subcarriers and cyclic prefix (CP) duration $T_{\mathrm{CP}}$ is considered. The reference signal is transmitted on $N_{\mathrm{P}}$ subcarriers, whose indices are described by $\mathcal{P}=\left\{p_{1}, \ldots, p_{N_{\mathrm{P}}}\right\}$ and $N_{\mathrm{B}}$ OFDM symbols are transmitted. We assume a narrowband signal model, i.e. $B / f_{\mathrm{c}} \ll \lambda_{\mathrm{c}} / D_{\max }$, where $B \approx \Delta f(\max (\mathcal{P})-\min (\mathcal{P}))$ is the signal bandwidth, $f_{c}$ is the carrier frequency, $\lambda_{\mathrm{c}}$ is the carrier wavelength and $D_{\max }$ is the largest of the Tx and Rx array apertures. The reference signal resource grid $\mathcal{R}$ comprises all resource elements at the time-frequency points $(p, b), p \in \mathcal{P}, b=0, \ldots, N_{\mathrm{B}}-1$. The transmitter uses a beam codebook $\left\{\boldsymbol{f}_{k}\right\}_{k=1}^{M_{\mathrm{T}}}$, where $M_{\mathrm{T}}$ is the number of beams in the codebook and $\left\|\boldsymbol{f}_{k}\right\|_{2}=1, \forall k$. The 
$k$-th beam is used on a subset $\mathcal{R}_{k}$ of resource elements (REs) $(p, b)$, with $\mathcal{R}_{k} \cap \mathcal{R}_{k^{\prime}}=\emptyset$ for $k \neq k^{\prime}$. The transmitted signal vector at the $p$-th subcarrier, $p \in \mathcal{P}$, of the $b$-th OFDM symbol, $b=0, \ldots, N_{\mathrm{B}}-1$, then is

$$
\boldsymbol{x}[p, b]=\lambda_{k}[p, b] \boldsymbol{f}_{k},(p, b) \in \mathcal{R}_{k},
$$

where

$$
\lambda_{k}[p, b]=\sqrt{P_{\text {tot }} q_{k} \gamma_{k}[p, b]} \mathrm{e}^{\mathrm{j} \beta_{k}[p, b]}
$$

is the symbol assigned to $f_{k}$ at the $p$-th subcarrier, $P_{\text {tot }}$ is the total Tx power (disregarding the power spent for the CP), $q_{k}$ is the fraction of $P_{\text {tot }}$ allocated to $\boldsymbol{f}_{k}$, with $\sum_{k=1}^{M_{\mathrm{T}}} q_{k}=1, \gamma_{k}[p, b]$ is the fraction of $q_{k}$ allocated to the $\operatorname{RE}(p, b)$, with $\sum_{(p, b) \in \mathcal{R}_{k}} \gamma_{k}[p, b]=1$, and $\beta_{k}[p, b]$ is the phase of $\lambda_{k}[p, b]$. The received signal is

$$
\boldsymbol{y}[p, b]=\boldsymbol{m}[p, b]+\boldsymbol{\eta}[p, b]
$$

where

$$
\begin{aligned}
& \boldsymbol{m}[p, b]=\sum_{l=0}^{L-1} h_{l} \mathrm{e}^{-\mathrm{j} \omega_{p} \tau_{l}} \boldsymbol{a}_{\mathrm{R}}\left(\theta_{\mathrm{R}, l}\right) \boldsymbol{a}_{\mathrm{T}}^{\mathrm{T}}\left(\theta_{\mathrm{T}, l}\right) \boldsymbol{x}[p, b], \\
& \boldsymbol{a}_{\mathrm{T}}\left(\theta_{\mathrm{T}, l}\right)=\left[\begin{array}{lll}
e^{\mathrm{j} \omega_{c} \tau_{\mathrm{T}, 1}\left(\theta_{\mathrm{T}, l}\right)}, & \ldots, & \mathrm{e}^{\mathrm{j} \omega_{c} \tau_{\mathrm{T}, N_{\mathrm{T}}}\left(\theta_{\mathrm{T}, l}\right)}
\end{array}\right]^{\mathrm{T}} \in \mathbb{C}^{N_{\mathrm{T}}}
\end{aligned}
$$

is the Tx array steering vector, with the Rx steering vector $\boldsymbol{a}_{\mathrm{R}}\left(\theta_{\mathrm{R}, l}\right)$ defined accordingly, $\omega_{p}=$ $2 \pi p \Delta f, \omega_{\mathrm{c}}=2 \pi f_{\mathrm{c}}, h_{l}$ is the gain of the $l$-th path and $\boldsymbol{\eta}[p, b] \sim \mathcal{N}_{\mathbb{C}}\left(\mathbf{0}, \sigma_{\eta}^{2} \boldsymbol{I}_{N_{\mathrm{R}}}\right)$ is the additive white Gaussian noise (AWGN). We write the signal model (11) as

$$
\boldsymbol{Y}_{b}=\sum_{l=0}^{L-1} h_{l} \boldsymbol{C}_{b}\left(\tau_{l}, \theta_{\mathrm{T}, l}, \theta_{\mathrm{R}, l}\right)+\boldsymbol{N}_{b}
$$

where

$$
\begin{aligned}
& \boldsymbol{C}_{b}\left(\tau_{l}, \theta_{\mathrm{T}, l}, \theta_{\mathrm{R}, l}\right)=\boldsymbol{a}_{\mathrm{R}}\left(\theta_{\mathrm{R}, l}\right) \boldsymbol{a}_{\mathrm{T}}^{\mathrm{T}}\left(\theta_{\mathrm{T}, l}\right) \boldsymbol{X}_{b} \operatorname{diag}\left(\boldsymbol{a}_{\tau}\left(\tau_{l}\right)\right) \in \mathbb{C}^{N_{\mathrm{R}} \times N_{\mathrm{P}}} \\
& \boldsymbol{a}_{\tau}(\tau)=\left[\mathrm{e}^{-\mathrm{j} \omega_{p_{1}} \tau}, \ldots, \mathrm{e}^{-\mathrm{j} \omega_{p_{N_{\mathrm{P}}}} \tau}\right]^{\mathrm{T}} \in \mathbb{C}^{N_{\mathrm{P}}} \\
& \boldsymbol{Y}_{b}=\left[\boldsymbol{y}\left[p_{1}, b\right], \ldots, \boldsymbol{y}\left[p_{N_{\mathrm{P}}}, b\right]\right] \in \mathbb{C}^{N_{\mathrm{R}} \times N_{\mathrm{P}}} \\
& \boldsymbol{X}_{b}=\left[\boldsymbol{x}\left[p_{1}, b\right], \ldots, \boldsymbol{x}\left[p_{N_{\mathrm{P}}}, b\right]\right] \in \mathbb{C}^{N_{\mathrm{T}} \times N_{\mathrm{P}}} \\
& \boldsymbol{N}_{b}=\left[\boldsymbol{\eta}\left[p_{1}, b\right], \ldots, \boldsymbol{\eta}\left[p_{N_{\mathrm{P}}}, b\right]\right] \in \mathbb{C}^{N_{\mathrm{R}} \times N_{\mathrm{P}}}
\end{aligned}
$$

Stacking the observations over $N_{\mathrm{B}}$ OFDM symbols we get

$$
\boldsymbol{Y}=\sum_{l=0}^{L-1} h_{l} \boldsymbol{C}\left(\tau_{l}, \theta_{\mathrm{T}, l}, \theta_{\mathrm{R}, l}\right)+\boldsymbol{N}
$$


where

$$
\begin{aligned}
\boldsymbol{Y} & =\left[\boldsymbol{Y}_{0}^{\mathrm{T}}, \ldots, \boldsymbol{Y}_{N_{\mathrm{B}}-1}^{\mathrm{T}}\right]^{\mathrm{T}} \\
\boldsymbol{C}\left(\tau, \theta_{\mathrm{T}}, \theta_{\mathrm{R}}\right) & =\left[\boldsymbol{C}_{0}^{\mathrm{T}}\left(\tau, \theta_{\mathrm{T}}, \theta_{\mathrm{R}}\right), \ldots, \boldsymbol{C}_{N_{\mathrm{B}}-1}^{\mathrm{T}}\left(\tau, \theta_{\mathrm{T}}, \theta_{\mathrm{R}}\right)\right]^{\mathrm{T}} \\
\boldsymbol{N} & =\left[\boldsymbol{N}_{0}^{\mathrm{T}}, \ldots, \boldsymbol{N}_{N_{\mathrm{B}}-1}^{\mathrm{T}}\right] .
\end{aligned}
$$

Through (4), (7)-(8) and (20), we can see that the observations $\boldsymbol{Y}$ depend on the position parameter vector $\boldsymbol{\nu}$, defined as

$$
\boldsymbol{\nu}=\left[\boldsymbol{p}_{\mathrm{R}}^{\mathrm{T}}, \alpha_{\mathrm{R}}, \epsilon_{\mathrm{clk}}, \boldsymbol{h}_{0}^{\mathrm{T}}, \boldsymbol{p}_{\mathrm{s}, 1}^{\mathrm{T}}, \boldsymbol{h}_{1}^{\mathrm{T}}, \ldots, \boldsymbol{p}_{\mathrm{s}, L-1}^{\mathrm{T}}, \boldsymbol{h}_{L-1}^{\mathrm{T}}\right]^{\mathrm{T}} \in \mathbb{R}^{4 L+2}
$$

with $\boldsymbol{h}_{l}=\left[\left|h_{l}\right|, \arg \left(h_{l}\right)\right]^{\mathrm{T}}$.

\section{Assumptions}

1) Reference signal structure: In this work we consider the case where Tx uses a fixed beam codebook $\boldsymbol{f}_{k}, k=1, \ldots, M_{\mathrm{T}}$. This does not only simplify the optimization task, but also might be a practical limitation in a $5 \mathrm{G}$ system, with devices using a predefined set of beams for transmission or reception.

We also assume that the resource allocation $\mathcal{R}_{k}$ among the codebook beams and the power allocation $\gamma_{k}[p, b]$ among assigned REs, are fixed and therefore, optimizing $\mathcal{R}_{k}$ is not in the scope of our reference signal optimization task. The problem of designing a waveform the has been addressed in [42]-[44], where the CRLB is optimized with respect to the resource allocation and additional constraints may be considered to avoid a unbalanced use of the spectrum.

2) Prior knowledge at $R x$ and $T x$ : In many cases the Tx might have prior knowledge on $\boldsymbol{\nu}$, based on prior estimation in the reverse link, map information and known geographical distribution of the users. The prior information is encoded by the joint probability density function (pdf) $p_{\nu}(\boldsymbol{\nu})$. In the following, we examine how the Tx can expoit the prior information, so as to improve the ability to localize the Rx.

The Rx, which aims to compute its position and orientation from the received signal, only has knowledge on the clock offset's distribution $p_{\epsilon_{\mathrm{clk}}}$, which we assume to be zero-mean Gaussian with variance $\sigma_{\mathrm{clk}}^{2}$. We note that $\sigma_{\mathrm{clk}}=0$ and $\sigma_{\mathrm{clk}} \rightarrow \infty$ correspond to perfect synchronization and asynchronous operation, respectively. 


\section{Position ERror Bound}

The achievable positioning accuracy of the Rx can be characterized in terms of the hybrid CRLB. For a parameter vector $\nu$ containing both deterministic and random paramters, the covariance matrix $\boldsymbol{C}$ of any unbiased estimator $\hat{\boldsymbol{\nu}}$ of $\boldsymbol{\nu}$ satisfies [45], [46]

$$
C-J_{\nu}^{-1} \geq 0
$$

where $\geq \mathbf{0}$ denotes positive semi-definiteness and $\boldsymbol{J}_{\boldsymbol{\nu}} \in \mathbb{R}^{(4 L+2) \times(4 L+2)}$ is the hybrid Fisher information matrix (FIM) of $\boldsymbol{\nu} . \boldsymbol{J}_{\boldsymbol{\nu}}$ is defined as

$$
\boldsymbol{J}_{\nu}=\boldsymbol{J}_{\nu}^{(\mathrm{p})}+\boldsymbol{J}_{\nu}^{(\mathrm{o})}
$$

where

$$
\boldsymbol{J}_{\boldsymbol{\nu}}^{(\mathrm{p})}=\mathbb{E}_{\boldsymbol{\nu}_{r}}\left[-D_{\nu}^{2} \ln p\left(\boldsymbol{\nu}_{r}\right)\right]
$$

accounts for the prior information and

$$
\boldsymbol{J}_{\nu}^{(\mathrm{o})}=\mathbb{E}_{\boldsymbol{Y}, \boldsymbol{\nu}_{r}}\left[-D_{\nu}^{2} \ln p(\boldsymbol{Y} \mid \boldsymbol{\nu})\right]
$$

accounts for the observation-related information, with $\nu_{r}$ representing the random parameters in $\nu$. As $\epsilon_{\mathrm{clk}}$ is the only parameter with prior information at the Rx, it is straightforward to find that, based on (24), the only non-zero entry of $J_{\nu}^{(\mathrm{p})}$ is

$$
\left[\boldsymbol{J}_{\nu}^{(\mathrm{p})}\right]_{4,4}=1 / \sigma_{\mathrm{clk}}^{2} \text {. }
$$

Since $\nu$ is observed under AWGN, the $(i, j)$-th entry of the $J_{\nu}^{(\text {o) }}$ is

$$
\left[\boldsymbol{J}_{\nu}^{(\mathrm{o})}\right]_{i, j}=\frac{2}{\sigma_{\eta}^{2}} \sum_{b=1}^{N_{\mathrm{B}}} \sum_{p \in \mathcal{P}} \mathfrak{R}\left\{\frac{\partial \boldsymbol{m}_{b}^{\mathrm{H}}[p]}{\partial v_{i}} \frac{\partial \boldsymbol{m}_{b}[p]}{\partial v_{j}}\right\} .
$$

Using (4), (12) and (30), we can see that $\boldsymbol{J}_{\nu}^{(\mathrm{o})}$ is independent of the value of $\epsilon_{\mathrm{clk}}$. The SPEB is defined as

$$
\mathrm{SPEB}=\operatorname{tr}\left(\boldsymbol{E}^{\mathrm{T}} \boldsymbol{J}_{\nu}^{-1} \boldsymbol{E}\right)
$$

where $\boldsymbol{E}=\left[\boldsymbol{e}_{1}, \boldsymbol{e}_{2}\right]$ and $\boldsymbol{e}_{i}$ is the $i$-th column of the identity matrix of the appropriate size. The position error bound (PEB) is defined as its square root. 


\section{Beam Power Allocation Optimization}

For the reference signal optimization, we make use of the assumption that with large bandwisth and number of antennas the paths are asymptotically orthogonal [9], [12]. We note that the SPEB is a function of

$$
\boldsymbol{\nu}^{\prime}=\left[\boldsymbol{p}_{\mathrm{R}}^{\mathrm{T}}, \alpha_{\mathrm{R}},\left|h_{0}\right|, \boldsymbol{p}_{\mathrm{s}, 1}^{\mathrm{T}},\left|h_{1}\right|, \ldots, \boldsymbol{p}_{\mathrm{s}, L-1}^{\mathrm{T}},\left|h_{L-1}\right|^{\mathrm{T}}\right]^{\mathrm{T}} \in \mathbb{R}^{3 L+1},
$$

that is, it is independent of the values of $\arg \left(h_{l}\right), l=1, \ldots, L-1$, and $\epsilon_{\mathrm{clk}}$. Also, due to the inner product of the derivatives in (30), we can observe (see (9), (10) and (12)) that $\boldsymbol{J}$ is independent of $\beta_{k}[p, b]$. In the following, we write $\boldsymbol{J}_{\boldsymbol{\nu}}=\boldsymbol{J}_{\boldsymbol{\nu}}\left(\boldsymbol{q}, \boldsymbol{\nu}^{\prime}\right)$, with $\boldsymbol{q}=\left[q_{1}, \ldots, q_{M_{\mathrm{T}}}\right] \in \mathbb{R}^{M_{\mathrm{T}}}$, to stress that $\boldsymbol{J}_{\boldsymbol{\nu}}$ is the hybrid FIM of $\boldsymbol{\nu}$, whose value depends on $\boldsymbol{q}$ and $\boldsymbol{\nu}^{\prime}$. Similarly, we write $\operatorname{SPEB}=\operatorname{SPEB}\left(\boldsymbol{q}, \boldsymbol{\nu}^{\prime}\right)$.

We study how the Tx can optimize the beam power allocation $\boldsymbol{q}$ using its prior knowledge on $\boldsymbol{\nu}^{\prime}$ so as to enable higher positioning accuracy at the Rx. We choose the expected SPEB (ESPEB)

$$
\operatorname{ESPEB}=\mathbb{E}_{\boldsymbol{\nu}^{\prime}}\left[\operatorname{SPEB}\left(\boldsymbol{q}, \boldsymbol{\nu}^{\prime}\right)\right]
$$

as the performance metric. The following proposed methods can be easily adapted for other objectives, such as $\max _{\boldsymbol{\nu}^{\prime}} \operatorname{SPEB}\left(\boldsymbol{q}, \boldsymbol{\nu}^{\prime}\right)$.

\section{A. Problem formulation}

The optimization problem in hand reads as:

$$
\min _{\boldsymbol{q}} \mathbb{E}_{\boldsymbol{\nu}^{\prime}}\left[\operatorname{SPEB}\left(\boldsymbol{q}, \boldsymbol{\nu}^{\prime}\right)\right] \text { s.t. } \boldsymbol{q} \geqslant \mathbf{0}, \mathbf{1}^{\mathrm{T}} \boldsymbol{q} \leq 1,
$$

where $\geqslant$ denotes element-wise inequality. In order to solve (34), one can employ a cubature rule [47], [48] with positive weights to approximate the expectation integral with a sum:

$$
\mathbb{E}_{\boldsymbol{\nu}^{\prime}}\left[\operatorname{SPEB}\left(\boldsymbol{q}, \boldsymbol{\nu}^{\prime}\right)\right] \approx \sum_{j=1}^{N_{\nu}^{\prime}} p_{j} \operatorname{SPEB}\left(\boldsymbol{q}, \boldsymbol{\nu}_{j}^{\prime}\right),
$$

where $\nu_{j}^{\prime}$ and $p_{j}>0, j=1, \ldots, N_{\nu^{\prime}}$ are the cubature points and their corresponding weights, with $N_{\nu^{\prime}}$ being the number of cubature points. $N_{\nu^{\prime}}$ is determined by the dimension of $\nu^{\prime}$ and the degree $r$ of the cubature ${ }^{1}$. The cubature points and their weights are determined by the pdf of $\nu^{\prime}$ and $r$. Then, (34) becomes

$$
\min _{\boldsymbol{q}} \sum_{j=1}^{N_{\boldsymbol{\nu}^{\prime}}} p_{j} \operatorname{SPEB}\left(\boldsymbol{q}, \boldsymbol{\nu}_{j}^{\prime}\right) \text { s.t. } \boldsymbol{q} \geqslant \mathbf{0}, \mathbf{1}^{\mathrm{T}} \boldsymbol{q} \leq 1 .
$$

\footnotetext{
${ }^{1} \mathrm{~A}$ cubature rule has degree $r$ if it is exact for a (multivariate) polynomial of degree $r$.
} 
In a similar fashion to [36], using the epigraph form of [36), we can show that it is equivalent to the following SDP:

$$
\begin{aligned}
\min _{\boldsymbol{q}, \boldsymbol{B}_{1}, \ldots, \boldsymbol{B}_{N_{\boldsymbol{\nu}^{\prime}}}} \sum_{j=1}^{N_{\boldsymbol{\nu}^{\prime}}} p_{j} \operatorname{tr}\left(\boldsymbol{B}_{j}\right) \quad \text { s.t. } & {\left[\begin{array}{cc}
\boldsymbol{B}_{j} & \boldsymbol{E}^{\mathrm{T}} \\
\boldsymbol{E} & \boldsymbol{J}\left(\boldsymbol{q}, \boldsymbol{\nu}_{j}^{\prime}\right)
\end{array}\right] \geq \mathbf{0}, j=1, \ldots, N_{\boldsymbol{\nu}^{\prime}} } \\
& \boldsymbol{q} \geqslant \mathbf{0}, \mathbf{1}^{\mathrm{T}} \boldsymbol{q} \leq 1
\end{aligned}
$$

where $\boldsymbol{B}_{j} \in \mathbb{R}^{2 \times 2}, j=1, \ldots, N_{\nu}$ are auxiliary variables of the SDP and $\geq$ denotes positive semidefiniteness. The positivity requirement on the cubature weights is imposed to ensure convexity of the objective in (37).

The optimal vector q obtained with (37) may indicate that a very low power should be allocated in the direction of the LOS path, which may lead to a missed detection of the LOS path at the Rx. This can be avoided by ensuring that the excitation on directions around the LOS path is at least a fraction $q_{\text {th }}$ of the excitation in any other direction. To this end, for a given confidence level $\kappa$, we define $\theta_{\mathrm{T}, l, \min }^{(\kappa)}$ and $\theta_{\mathrm{T}, l, \max }^{(\kappa)}$ as the minimum and maximum AODs corresponding to the two-dimensional (2D) Rx locations $(l=0)$ or scatterer/reflector locations $(l=1, \ldots, L-1)$ in the $\kappa$-confidence ellipse of the respective marginal. With a uniform grid of $N_{\theta}$ possible AODs $\theta_{\mathrm{T}, l, m}$ within the interval $\left[\theta_{\mathrm{T}, l, \min }^{(\kappa)}, \theta_{\mathrm{T}, l, \max }^{(\kappa)}\right]$

$$
\theta_{\mathrm{T}, l, m}^{(\kappa)}=\theta_{\mathrm{T}, l, \min }^{(\kappa)}+\frac{m-1}{N_{\theta}-1} \theta_{\mathrm{T}, l, \max }^{(\kappa)}, m=1, \ldots, N_{\theta},
$$

we define the excitation matrix $\boldsymbol{A}_{l} \in \mathbb{R}^{N_{\theta} \times M_{\mathrm{T}}}$ for the $l$-th path as

$$
\left[\boldsymbol{A}_{l}\right]_{m, k}=\left|\boldsymbol{a}_{\mathrm{T}}^{\mathrm{T}}\left(\theta_{\mathrm{T}, l, m}^{(\kappa)}\right) \boldsymbol{f}_{k}\right|^{2} .
$$

Finally, the excitation vector for the possible AODs of the $l$-th path is $\boldsymbol{A}_{l} \boldsymbol{q}$. Finally, the vector with the excitation of the possible AODs associated with the $l$-th path is $\boldsymbol{A}_{l} \boldsymbol{q}$. We augment (37) with the following linear constraints:

$$
\boldsymbol{A}_{0} \boldsymbol{q} \geqslant q_{\mathrm{th}}\|\boldsymbol{A} \boldsymbol{q}\|_{\infty} \mathbf{1}_{N_{\theta}},
$$

where $\boldsymbol{A}=\left[\boldsymbol{A}_{0}^{\mathrm{T}}, \ldots, \boldsymbol{A}_{L-1}^{\mathrm{T}}\right]^{\mathrm{T}}$. We note that the constraints 40 can be equivalently expressed as

$$
\boldsymbol{A}_{0} \boldsymbol{q} \geqslant q_{\mathrm{th}} e_{\max } \mathbf{1}_{N_{\theta}}, \quad \boldsymbol{A q} \preccurlyeq e_{\max } \mathbf{1}_{L N_{\theta}},
$$

with $e_{\max }$ being an auxiliary optimization variable. 
The main challenge with the approach described above is that $p_{\nu}$ is a multidimensional pdf. The number of auxiliary matrices $\boldsymbol{B}_{j}$ and corresponding positive semidefiniteness (PSD) constraints in (37) is equal to the number of cubature points. For known cubature rules [47], the number of points is lower bounded by $(3 L+1)^{(r-1) / 2}$, which could result in very high complexity for our optimization task, as the integrand is highly non-linear and a rule with $r \geq 5$ is required for an accurate approximation.

\section{B. Low-complexity sub-optimal solution}

1) Dimensionality reduction: A way to circumvent the dimensionality challenge is to use a surrogate function which involves the expectation over a smaller set of parameters. To this end, we first note that $\boldsymbol{e}_{i}^{\mathrm{T}} \boldsymbol{J}^{-1} \boldsymbol{e}_{i}, i=1,2$, is a convex function of $\boldsymbol{J}$ and so is the SPEB as a sum of convex functions. Splitting $\nu^{\prime}$ into any couple of vectors $\nu_{1}$ and $\nu_{2}$, we can write

$$
\begin{aligned}
\mathbb{E}_{\boldsymbol{\nu}}[\operatorname{SPEB}(\boldsymbol{q}, \boldsymbol{\nu})] & =\mathbb{E}_{\boldsymbol{\nu}}\left[\operatorname{tr}\left(\boldsymbol{E}^{\mathrm{T}} \boldsymbol{J}^{-1}\left(\boldsymbol{q}, \boldsymbol{\nu}^{\prime}\right) \boldsymbol{E}\right)\right]=\mathbb{E}_{\boldsymbol{\nu}_{1}}\left[\mathbb{E}_{\boldsymbol{\nu}_{2} \mid \boldsymbol{\nu}_{1}}\left[\operatorname{tr}\left(\boldsymbol{E}^{\mathrm{T}} \boldsymbol{J}^{-1}\left(\boldsymbol{q}, \boldsymbol{\nu}_{1}, \boldsymbol{\nu}_{2}\right) \boldsymbol{E}\right)\right]\right] \\
& \stackrel{(a)}{\geq} \mathbb{E}_{\boldsymbol{\nu}_{1}}\left[\operatorname{tr}\left(\boldsymbol{E}^{\mathrm{T}}\left(\mathbb{E}_{\boldsymbol{\nu}_{2} \mid \boldsymbol{\nu}_{1}}\left[\boldsymbol{J}\left(\boldsymbol{q}, \boldsymbol{\nu}_{1}, \boldsymbol{\nu}_{2}\right)\right]\right)^{-1} \boldsymbol{E}\right)\right]
\end{aligned}
$$

where (a) follows from Jensen's inequality. We choose $\boldsymbol{\nu}_{1}=\left[\boldsymbol{p}_{\mathrm{R}}^{\mathrm{T}}, \boldsymbol{p}_{\mathrm{s}, 1}^{\mathrm{T}}, \ldots, \boldsymbol{p}_{\mathrm{s}, L-1}^{\mathrm{T}}\right]^{\mathrm{T}}$, as the position parameters are the ones determining the AODs, which in turn determine which beams are relevant or not. One could optimize the lower bound on the ESPEB in (42), as described in (34)-(37), but the number of cubature points $N_{\boldsymbol{\nu}^{\prime}}$ is still lower bounded by $(2 L)^{(r-1) / 2}$.

2) Power allocation as a weighted sum of per-path power allocation vectors: Our aim is to reduce the complexity of the optimization problem in hand. We accomplish this by taking the following heuristic approach: we compute a power allocation vector $\boldsymbol{q}_{l}, l=0, \ldots, L-1$, considering the uncertainty regarding each path separately and then weight the resulting power allocation vectors in order to minimize a lower bound on the ESPEB.

More specifically, for the power allocation vector $\boldsymbol{q}_{0}$ we consider only the LOS path and neglect the NLOS paths and solve

$$
\begin{aligned}
& \boldsymbol{q}_{0}=\underset{\boldsymbol{q}}{\operatorname{argmin}} \mathbb{E}_{\boldsymbol{p}_{\mathrm{R}}}\left[\operatorname{tr}\left(\boldsymbol{E}^{\mathrm{T}}\left(\mathbb{E}_{\left|h_{0}\right|, \alpha_{\mathrm{R}} \mid \boldsymbol{p}_{\mathrm{R}}}\left[\boldsymbol{J}_{\boldsymbol{\nu}_{\mathrm{LOS}}}\left(\boldsymbol{q}, \boldsymbol{p}_{\mathrm{R}}, \alpha_{\mathrm{R}},\left|h_{0}\right|\right)\right]\right)^{-1} \boldsymbol{E}\right)\right] \quad \text { s.t. } \boldsymbol{A}_{0} \boldsymbol{q} \geqslant q_{\mathrm{th}, \mathrm{LOS}}\left\|\boldsymbol{A}_{0} \boldsymbol{q}\right\|_{\infty} \mathbf{1}_{N_{\theta}}, \\
& \boldsymbol{q} \geqslant \mathbf{0}, \mathbf{1}^{\mathrm{T}} \boldsymbol{q} \leq 1,
\end{aligned}
$$

where $\boldsymbol{J}_{\nu_{\mathrm{LOS}}}$ represents the FIM for the parameter vector $\boldsymbol{\nu}_{\mathrm{LOS}}=\left[\boldsymbol{p}_{\mathrm{R}}^{\mathrm{T}}, \alpha_{\mathrm{R}}, \epsilon_{\mathrm{clk}}, \boldsymbol{h}_{0}^{\mathrm{T}}\right]^{\mathrm{T}}$. Similarly to (40), the first constraint in (43) limits the ratio of power spent among possible LOS directions, with $q_{\text {th,LOS }}$ being the corresponding minimum ratio. For the gain of the LOS path it is natural 
that $p\left(\boldsymbol{h}_{0} \mid \boldsymbol{p}_{\mathrm{R}}\right)=p\left(\boldsymbol{h}_{0} \mid d_{0}\right)$, with $d_{0}=\left\|\boldsymbol{p}_{\mathrm{R}}\right\|_{2}$, i.e. the distribution of the gain depends only on the Tx-Rx distance, and the integration over the radial component $d_{0}$ and the angular component $\theta_{\mathrm{T}, 0}$ of $\boldsymbol{p}_{\mathrm{R}}$ can be carried out separately. Then, as shown in the Appendix, we can reformulate (43) as an SDP using a one-dimensional (1D) quadrature rule for the approximation of the expectation integral over $\theta_{\mathrm{T}, 0}$.

For the power allocation vector $\boldsymbol{q}_{l}$ we consider only the $l$-th NLOS path and assume that the Rx position and orientation are known and equal to their mean values $\bar{p}_{\mathrm{R}}$ and $\bar{\alpha}_{\mathrm{R}}$. This is basically a bistatic radar setup, where the goal is the estimation of the point of incidence. Therefore, we obtain $\boldsymbol{q}_{l}$ by solving

$$
\boldsymbol{q}_{l}=\underset{\boldsymbol{q}}{\operatorname{argmin}} \mathbb{E}_{\boldsymbol{s}_{\mathrm{s}, l}}\left[\operatorname{tr}\left(\boldsymbol{E}^{\mathrm{T}}\left(\mathbb{E}_{\mid h_{l} \| \boldsymbol{p}_{\mathrm{s}, l}}\left[\boldsymbol{J}_{\mathrm{NLOS}, l}\left(\boldsymbol{q}, \boldsymbol{p}_{\mathrm{s}, l},\left|h_{l}\right|\right)\right]\right)^{-1} \boldsymbol{E}\right)\right] \quad \text { s.t. } \boldsymbol{q} \geqslant \mathbf{0}, \mathbf{1}^{\mathrm{T}} \boldsymbol{q} \leq 1,
$$

where $\boldsymbol{J}_{\mathrm{NLOS}, l}$ represent the FIM for the parameter vector $\boldsymbol{\nu}_{\mathrm{NLOS}, l}=\left[\boldsymbol{p}_{\mathrm{s}, l}^{\mathrm{T}}, \epsilon_{\mathrm{clk}}, \boldsymbol{h}_{l}^{\mathrm{T}}\right]^{\mathrm{T}}$. Problem (44) can be solved employing a 2D cubature on $\boldsymbol{p}_{\mathrm{s}, l}$.

Finally, we compute the optimal weights $\boldsymbol{w} \in \mathbb{R}^{L}$ of $\boldsymbol{q}_{l}, l=0, \ldots, L-1$, by minimizing an approximate lower bound on the ESPEB, obtained similarly to 42]:

$$
\begin{gathered}
\boldsymbol{w}=\underset{\boldsymbol{w}^{\prime}}{\operatorname{argmin}} \mathbb{E}_{\boldsymbol{p}_{\mathrm{R}}}\left[\operatorname{tr}\left(\boldsymbol{E}^{\mathrm{T}} \boldsymbol{J}^{-1}\left(\boldsymbol{Q} \boldsymbol{w}^{\prime}, \overline{\boldsymbol{\nu}}\right) \boldsymbol{E}\right)\right] \quad \text { s.t. } \boldsymbol{A}_{0} \boldsymbol{Q} \boldsymbol{w}^{\prime} \geqslant q_{\mathrm{th}}\left\|\boldsymbol{A} \boldsymbol{Q} \boldsymbol{w}^{\prime}\right\|_{\infty} \mathbf{1}_{N_{\theta}} \\
\boldsymbol{Q} \boldsymbol{w}^{\prime} \geqslant \mathbf{0}, \mathbf{1}^{\mathrm{T}} \boldsymbol{Q} \boldsymbol{w}^{\prime} \leq 1,
\end{gathered}
$$

where, in order to further reduce the computational load, we have replaced $\mathbb{E}_{\boldsymbol{\nu} \mid \boldsymbol{p}_{\mathrm{R}}}\left[\boldsymbol{J}\left(\boldsymbol{Q} \boldsymbol{w}^{\prime}, \boldsymbol{\nu}\right)\right]$ with its approximation $\boldsymbol{J}\left(\boldsymbol{Q} \boldsymbol{w}^{\prime}, \overline{\boldsymbol{\nu}}\right)$, with $\overline{\boldsymbol{\nu}}=\mathbb{E}_{\boldsymbol{\nu} \mid \boldsymbol{p}_{\mathrm{R}}}[\boldsymbol{\nu}]$ and $\boldsymbol{Q}=\left[\boldsymbol{q}_{0}, \ldots, \boldsymbol{q}_{L-1}\right]$. Finally, the beam power allocation vector is $\boldsymbol{q}=\boldsymbol{Q w}$.

\section{Channel and Position Estimation}

In this section we present a novel algorithm for $\mathrm{Rx}$ position, orientation and clock offset estimation. In the first step of the algorithm a gridless parameter estimation algorithm based on [41] is employed to recover the number paths and their respective TOAs, AODs and AOAs. In the second step, the recovered channel parameters are mapped to the position parameter vector $\nu$. 


\section{A. Channel parameter estimation}

For our positioning purposes, we are not merely interested in denoising $\boldsymbol{Y}$, but we would like to recover the number of paths, along with their respective gains, TOAs, AODs and AOAs. Hence, we aim to solve the following optimization problem:

$$
\min _{L^{\prime},\left\{\tau_{l}, \theta_{\mathrm{T}, l}, \theta_{\mathrm{R}, l}, h_{l}\right\}_{l=0}^{L^{\prime}-1}} \Lambda(\boldsymbol{R})+\chi\|\boldsymbol{h}\|_{1}
$$

where

$$
\Lambda(\boldsymbol{R})=\frac{1}{2}\|\boldsymbol{R}\|_{\mathrm{F}}^{2}
$$

is the loss function,

$$
\boldsymbol{R}=\boldsymbol{Y}-\sum_{l=0}^{L^{\prime}-1} h_{l} \boldsymbol{C}\left(\tau_{l}, \theta_{\mathrm{T}, l} \theta_{\mathrm{R}, l}\right)
$$

is the residual, $\chi$ is a regularization parameter and $\boldsymbol{h}=\left[h_{0}, \ldots, h_{L^{\prime}-1}\right]^{\mathrm{T}}$. The penalty term $\|\boldsymbol{h}\|_{1}$ is included to make the channel representation more parsimonious; otherwise the number of detected paths could grow arbitrarily so as to minimize the objective. As usual in sparse recovery setups, instead of a non-convex L0 norm penalty term, we use the L1 norm. We solve problem (46] using the algorithmic framework of [41], termed as Alternating Descent Conditional Gradient Method (ADCGM), which is described in Alg. 1. We note that, for notational brevity, in (46)-(48) and in the following, we write $\boldsymbol{R}$ instead of $\boldsymbol{R}\left(L^{\prime},\left\{\tau_{l}, \theta_{\mathrm{T}, l}, \theta_{\mathrm{R}, l}\right\}_{l=0}^{L^{\prime}-1}\right)$. Also, the residual at iteration $i$ is denoted as $\boldsymbol{R}_{i}$ and the TOAs of the detected paths are stacked in the vector $\tau^{(i)}=\left[\tau_{0}^{(i)}, \ldots, \tau_{L^{(i)}-1}^{(i)}\right] \in \mathbb{R}^{L^{(i)}}$, where $L^{(i)}$ is the number of detected paths at iteration $i$. The parameter vectors $\boldsymbol{\theta}_{\mathrm{T}}^{(i)}$ and $\boldsymbol{\theta}_{\mathrm{R}}^{(i)}$ are defined accordingly. The maximum number of iterations is $L_{\max }$ and at each iteration a new path can be detected (Step 2) or previously detected paths can be dropped (Step 4(b)). In the following, we describe steps 2 and 4 in detail.

1) Detection of a new potential path (Step 2): In order to get the next potential path we have to solve (49), which is non-convex and can be solved by discretizing the three-dimensional (3D) parameter space $\left[0, T_{\mathrm{CP}}\right] \times[-\pi, \pi) \times[-\pi, \pi)$ to get an $N_{\tau} \times N_{\theta_{\mathrm{T}}} \times N_{\theta_{\mathrm{R}}}$-dimensional grid $\mathcal{G}$.

After computing the new potential source, we compare the correspoding objective with a predefined threshold $\zeta_{1}>0$, which is a function of the noise variance $\sigma_{\eta}^{2}$, the reference signal $\boldsymbol{X}$ and the desired false alarm probability $P_{\mathrm{fa}}$. 


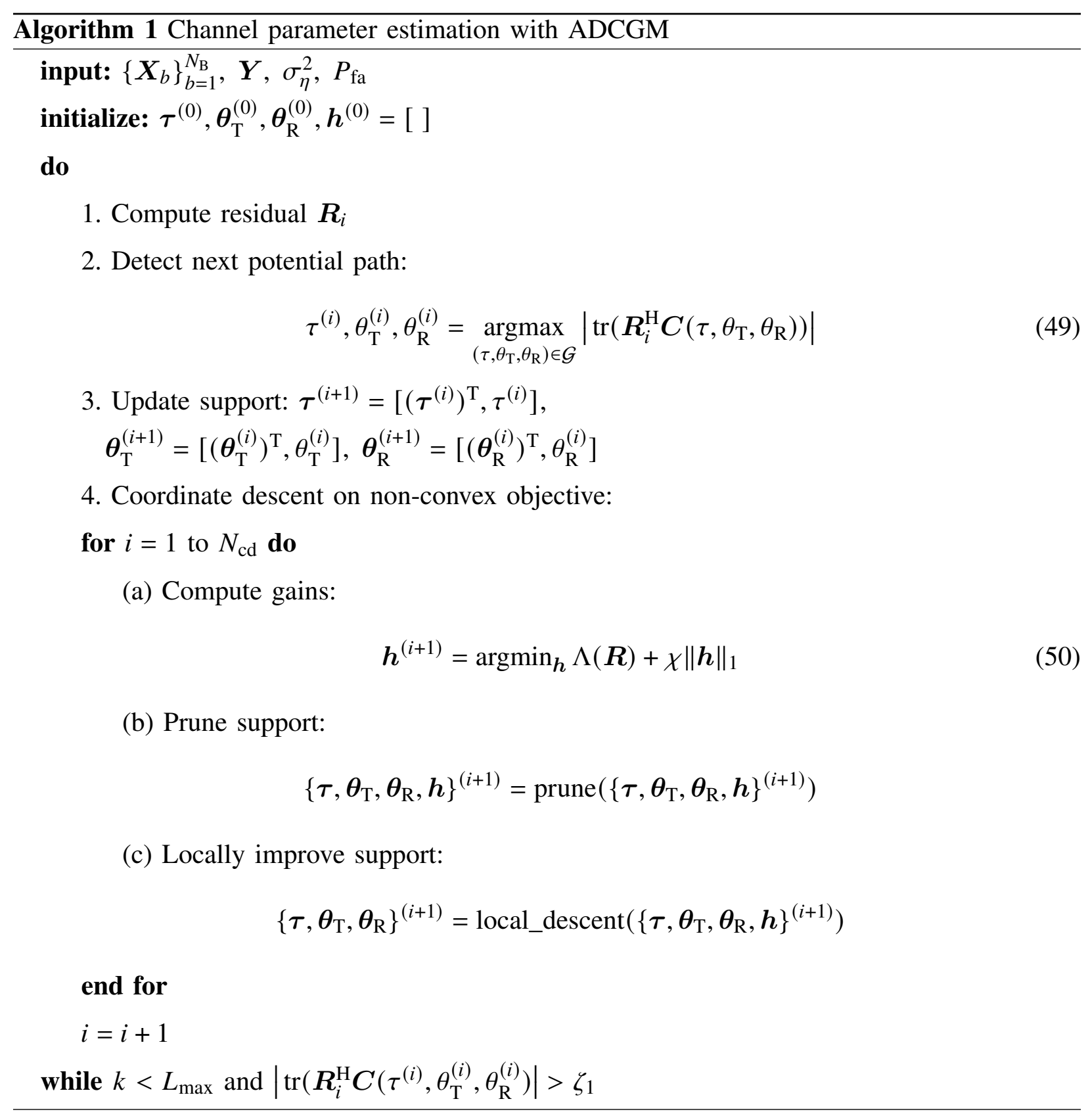

2) Coordinate descent (Step 4): In this algorithmic step we iteratively perform 3 sub-steps for a fixed number of $N_{\mathrm{cd}}$ iterations:

(a) We update the gains solving [50, keeping the other path parameters fixed. The regularization parameter $\chi$ determines the accuracy-sparsity trade-off.

(b) We prune the paths whose gain is effectively zero: the $l$-th path is pruned if $\left|h_{l}\right|^{2} / \zeta_{2}<$ $\max _{l=0, \ldots, L^{(i)}-1}\left|h_{l}\right|^{2}$, where $0<\zeta_{2} \ll 1$.

(c) For the local descent step we perform truncated Newton steps for each path and each 
parameter sequentially:

$$
\begin{aligned}
& \tau_{l}^{(i+1)} \leftarrow \tau_{l}^{(i+1)}-\operatorname{sgn}\left(\partial \Lambda / \partial \tau_{l}^{(i+1)}\right) s_{\tau, l}^{(i+1)} \\
& \theta_{\mathrm{T}, l}^{(i+1)} \leftarrow \theta_{\mathrm{T}, l}^{(i+1)}-\operatorname{sgn}\left(\partial \Lambda / \partial \theta_{\mathrm{T}, l}^{(i+1)}\right) s_{\theta_{\mathrm{T}}, l}^{(i+1)} \\
& \theta_{\mathrm{R}, l}^{(i+1)} \leftarrow \theta_{\mathrm{R}, l}^{(i+1)}-\operatorname{sgn}\left(\partial \Lambda / \partial \theta_{\mathrm{R}, l}^{(i+1)}\right) s_{\theta_{\mathrm{R}}, l}^{(i+1)}
\end{aligned}
$$

where

$$
\begin{aligned}
& s_{\tau, l}^{(i+1)}=\min \left(\left|\left(\partial^{2} \Lambda /\left(\partial \tau_{l}^{(i+1)}\right)^{2}\right)^{-1} \partial \Lambda / \partial \tau_{l}^{(i+1)}\right|, \frac{N_{\mathrm{CP}} T_{\mathrm{s}}}{2\left(N_{\tau}-1\right)}\right) \\
& s_{\theta_{\mathrm{T}}, l}^{(i+1)}=\min \left(\left|\left(\partial^{2} \Lambda /\left(\partial \theta_{\mathrm{T}, l}^{(i+1)}\right)^{2}\right)^{-1} \partial \Lambda / \partial \theta_{\mathrm{T}, l}^{(i+1)}\right|, \frac{\pi}{N_{\theta_{\mathrm{T}}-1}}\right) \\
& s_{\theta_{\mathrm{R}}, l}^{(i+1)}=\min \left(\left|\left(\partial^{2} \Lambda /\left(\partial \theta_{\mathrm{R}, l}^{(i+1)}\right)^{2}\right)^{-1} \partial \Lambda / \partial \theta_{\mathrm{R}, l}^{(i+1)}\right|, \frac{\pi}{N_{\theta_{\mathrm{R}}-1}}\right)
\end{aligned}
$$

are the step sizes, with $T_{\mathrm{s}}=N \Delta f$. We note that we limit the maximum step size for each of the parameters to be equal to half of the corresponding grid bin size, in order to avoid convergence problems near inflection points of the loss function.

\section{B. Mapping to position parameters}

Having an estimate $\hat{\tilde{\boldsymbol{\nu}}}$ of the channel parameter vector $\tilde{\boldsymbol{\nu}}$ defined as

$$
\tilde{\boldsymbol{\nu}}=\left[\tau_{0}, \theta_{\mathrm{T}, 0}, \theta_{\mathrm{R}, 0}, \ldots, \tau_{\hat{L}-1}, \theta_{\mathrm{T}, \hat{L}-1}, \theta_{\mathrm{R}, \hat{L}-1}\right]^{\mathrm{T}},
$$

where $\hat{L}$ is the estimated number of paths, and choosing the strongest path as the LOS path, we estimate the position parameter vector $\nu$ employing the EXIP as in [13], with a slight modification to include the prior information on the clock offset. To this end, we intend to solve

$$
\underset{\boldsymbol{\nu}}{\operatorname{argmin}}(\hat{\tilde{\boldsymbol{\nu}}}-f(\boldsymbol{\nu}))^{\mathrm{T}} \boldsymbol{J}_{\hat{\boldsymbol{\nu}}}(\hat{\tilde{\boldsymbol{\nu}}}-f(\boldsymbol{\nu}))+\left(\epsilon_{\mathrm{clk}} / \sigma_{\mathrm{clk}}\right)^{2},
$$

where $\boldsymbol{J}_{\hat{\boldsymbol{\nu}}}$ is the channel parameter FIM and $f: \mathbb{R}^{2 \hat{L}+2} \rightarrow \mathbb{R}^{3 \hat{L}}$ is the mapping from position to channel parameters, determined by (4), (7)-(8).

We note that false alarms, that is falsely detected paths, can have severe impact on position estimation. Therefore, we apply the following two criteria to filter them out:

- A single-bounce NLOS path and a LOS path always form a triangle, as can be seen in Fig. 1. Therefore, for the formation of a triangle to be possible, a single-bounce NLOS path must satisfy

$$
\Delta \theta_{\mathrm{T}, l} \cdot \Delta \theta_{\mathrm{R}, l}<0, l=1, \ldots, \hat{L}-1,
$$


where $\Delta \theta_{\mathrm{T}, l}=\theta_{\mathrm{T}, l}-\theta_{\mathrm{T}, 0}$ and $\Delta \theta_{\mathrm{R}, l}=\theta_{\mathrm{R}, l}-\theta_{\mathrm{R}, 0}$, with $\Delta \theta_{\mathrm{T}, l}$ and $\Delta \theta_{\mathrm{R}, l} \in[-\pi, \pi)$. Therefore if the $l$-th path, $l=1, \ldots, \hat{L}-1$, does not satisfy (56), it is dropped.

- Combined with the LOS path, each NLOS path can provide an estimate of $\epsilon_{\mathrm{clk}}$ :

$$
\epsilon_{\mathrm{clk}, l}=\frac{\tau_{l} \sin \left(\Delta \theta_{\mathrm{R}, l}-\Delta \theta_{\mathrm{T}, l}\right)-\tau_{0}\left(\sin \left(\Delta \theta_{\mathrm{R}, l}\right)-\sin \left(\Delta \theta_{\mathrm{T}, l}\right)\right)}{\sin \left(\Delta \theta_{\mathrm{R}, l}-\Delta \theta_{\mathrm{T}, l}\right)-\left(\sin \left(\Delta \theta_{\mathrm{R}, l}\right)-\sin \left(\Delta \theta_{\mathrm{T}, l}\right)\right)},
$$

With $\zeta_{3, a}>0$ and $\zeta_{3, b}>0$ being predefined probability thresholds for $\epsilon_{\mathrm{clk}}$ values, if $p\left(\epsilon_{\mathrm{clk}, l}\right)<\zeta_{3, a}$ or $p\left(\epsilon_{\mathrm{clk}, l}\right)<\zeta_{3, b} p_{\mathrm{clk}, \max }$, the path is filtered out, with $p_{\mathrm{clk}, \max }=\max _{l=1, \ldots, \hat{L}-1} p\left(\epsilon_{\mathrm{clk}, l}\right)$.

Replacing $\hat{\tilde{\boldsymbol{\nu}}}$ with $\hat{\tilde{\boldsymbol{\nu}}}^{\prime}$, which contains only the remaining paths, we solve (55) with the Levenberg-Marquardt algorthm [49], [50]. For the initial point $\boldsymbol{\nu}^{(0)}$ we compute

$$
\begin{aligned}
\epsilon_{\mathrm{clk}}^{(0)} & =\frac{\sum_{l}\left|h_{l}\right|^{2} \epsilon_{\mathrm{clk}, l}}{\sum_{l}\left|h_{l}\right|^{2}} \\
\boldsymbol{p}_{\mathrm{R}}^{(0)} & =c\left(\tau_{0}-\epsilon_{\mathrm{clk}}^{(0)}\right) \boldsymbol{u}\left(\theta_{\mathrm{T}, 0}\right) \\
\alpha_{\mathrm{R}}^{(0)} & =\theta_{\mathrm{T}, 0}+\pi-\theta_{\mathrm{R}, 0} \\
\boldsymbol{p}_{\mathrm{s}, l}^{(0)} & =\frac{\tan \left(\theta_{\mathrm{R}, l}+\alpha_{\mathrm{R}}^{(0)}\right) p_{\mathrm{R}, x}^{(0)}-p_{\mathrm{R}, y}^{(0)}}{\tan \left(\theta_{\mathrm{R}, l}+\alpha_{\mathrm{R}}^{(0)}\right) \cos \theta_{\mathrm{T}, l}-\sin \theta_{\mathrm{T}, l}} \boldsymbol{u}\left(\theta_{\mathrm{T}, l}\right), l=1, \ldots, \hat{L}^{\prime},
\end{aligned}
$$

where $\hat{L}^{\prime}$ is the number of remaining estimated paths.

\section{NUMERICAL RESULTS}

\section{A. Simulation setup}

1) Geometric setup and prior information at the Tx: For the evaluation of the power allocation and the position estimation algorithms we consider the setup shown in Fig. 2. The Tx is equipped with a ULA with $N_{\mathrm{T}}=32$ antennas. In order to be able to discriminate all possible AOAs, the Rx has a UCA with $N_{\mathrm{R}}=16$ antennas. With the Rx being equipped with a UCA, the SPEB is independent of the orientation $\alpha_{\mathrm{R}}$.

We consider NLOS paths resulting from single-bounce reflections. The phases of the complex path gains are uniformly distributed over $[-\pi, \pi)$ and their magnitudes are given by

$$
\left|h_{l}\right|= \begin{cases}c /\left(4 \pi f_{\mathrm{c}}\left\|\boldsymbol{p}_{\mathrm{R}}\right\|_{2}\right), & l=0, \\ \sqrt{\rho_{l}} c /\left(4 \pi f_{\mathrm{c}}\left(\left\|\boldsymbol{p}_{\mathrm{s}, l}\right\|_{2}+\left\|\boldsymbol{p}_{\mathrm{R}}-\boldsymbol{p}_{\mathrm{s}, l}\right\|_{2}\right)\right), & l \neq 0,\end{cases}
$$




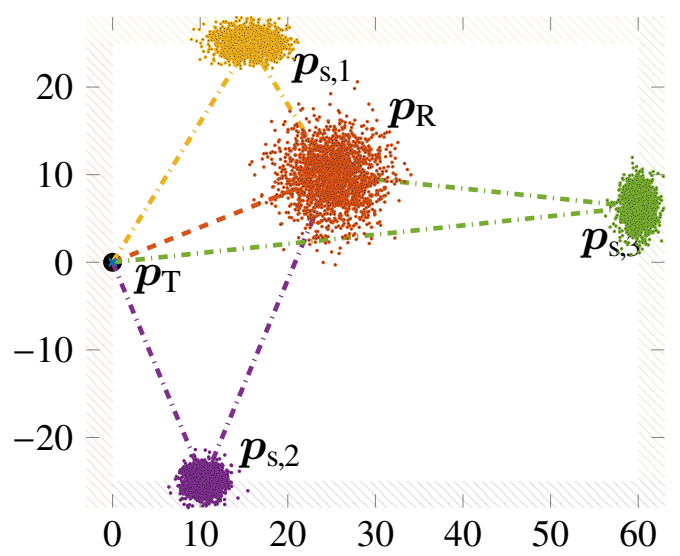

Fig. 2. Prior knowledge at the Tx for simulation results.

where $\rho_{l}$ is the reflection coefficient and $\lambda_{\mathrm{c}}=c / f_{\mathrm{c}}$. The prior knowledge at the Tx is described by $\mathcal{N}(\boldsymbol{\mu}, \boldsymbol{C})$, where

$$
\begin{aligned}
\boldsymbol{\mu} & =\left[\overline{\boldsymbol{p}}_{\mathrm{R}}^{\mathrm{T}}, \overline{\boldsymbol{p}}_{\mathrm{s}, 1}^{\mathrm{T}}, \bar{\rho}, \overline{\boldsymbol{p}}_{\mathrm{s}, 2}^{\mathrm{T}}, \bar{\rho}, \overline{\boldsymbol{p}}_{\mathrm{s}, 3}^{\mathrm{T}}, \bar{\rho}\right]^{\mathrm{T}} \in \mathbb{R}^{11} \\
\boldsymbol{C} & =\left[\begin{array}{ccccccc}
\boldsymbol{C}_{0,0} & \boldsymbol{C}_{0,1} & \mathbf{0} & \boldsymbol{C}_{0,2} & \mathbf{0} & \boldsymbol{C}_{0,3} & \mathbf{0} \\
\boldsymbol{C}_{0,1}^{\mathrm{T}} & \boldsymbol{C}_{1,1} & \mathbf{0} & \mathbf{0} & \mathbf{0} & \mathbf{0} & \mathbf{0} \\
\mathbf{0} & \mathbf{0} & \sigma_{\rho}^{2} & \mathbf{0} & 0 & \mathbf{0} & 0 \\
\boldsymbol{C}_{0,2}^{\mathrm{T}} & \mathbf{0} & \mathbf{0} & \boldsymbol{C}_{2,2} & \mathbf{0} & \mathbf{0} & \mathbf{0} \\
\mathbf{0} & \mathbf{0} & 0 & \mathbf{0} & \sigma_{\rho}^{2} & \mathbf{0} & 0 \\
\boldsymbol{C}_{0,3}^{\mathrm{T}} & \mathbf{0} & \mathbf{0} & \mathbf{0} & \mathbf{0} & \boldsymbol{C}_{3,3} & \mathbf{0} \\
\mathbf{0} & \mathbf{0} & 0 & \mathbf{0} & 0 & \mathbf{0} & \sigma_{\rho}^{2}
\end{array}\right] \in \mathbb{R}^{11 \times 11}
\end{aligned}
$$

with

$$
\begin{aligned}
\overline{\boldsymbol{p}}_{\mathrm{R}} & =\left[\begin{array}{c}
25 \\
10
\end{array}\right] \mathrm{m}, \boldsymbol{C}_{0,0}=4 / \sqrt{2} \boldsymbol{I}_{2} \mathrm{~m}^{2} \\
\overline{\boldsymbol{p}}_{\mathrm{s}, 1} & =\left[\begin{array}{c}
15.63 \\
25
\end{array}\right] \mathrm{m}, \boldsymbol{C}_{1,1}=\left[\begin{array}{cc}
3.48 & 0 \\
0 & 1
\end{array}\right] \mathrm{m}^{2}, \boldsymbol{C}_{0,1}=\left[\begin{array}{cc}
4.45 & 0 \\
0 & 0
\end{array}\right] \mathrm{m}^{2} \\
\overline{\boldsymbol{p}}_{\mathrm{s}, 2} & =\left[\begin{array}{c}
10.42 \\
-25
\end{array}\right] \mathrm{m}, \boldsymbol{C}_{2,2}=\left[\begin{array}{cc}
1.34 & 0 \\
0 & 1
\end{array}\right] \mathrm{m}^{2}, C_{0,2}=\left[\begin{array}{cc}
1.64 & 0 \\
0 & 0
\end{array}\right] \mathrm{m}^{2} \\
\overline{\boldsymbol{p}}_{\mathrm{s}, 3} & =\left[\begin{array}{c}
60 \\
6.32
\end{array}\right] \mathrm{m}, C_{3,3}=\left[\begin{array}{cc}
1 & 0 \\
0 & 2.31
\end{array}\right] \mathrm{m}^{2}, C_{0,3}=\left[\begin{array}{ll}
0 & 0 \\
0 & 3.24
\end{array}\right] \mathrm{m}^{2}
\end{aligned}
$$




$$
\bar{\rho}=-10 \mathrm{~dB}, \sigma_{\rho}=4 \mathrm{~dB} .
$$

Samples from this distribution are depicted in Fig. 2 .

2) System Parameters: For the waveform we set $f_{\mathrm{c}}=38 \mathrm{GHz}, N=64, N_{\mathrm{B}}=10, \mathcal{P}=$ $\{-31, \ldots,-1,1 \ldots, 31\}$ and $\Delta f(\max (\mathcal{P})-\min (\mathcal{P}))(\approx B)=120 \mathrm{MHz}$. The resources are assigned to the beams in an interleaved and staggered manner, i.e. $\mathcal{R}_{k}=\left\{\left(k+b+i M_{\mathrm{T}}, b\right) \mid i \in\right.$ $\left.\mathbb{Z}, b=1, \ldots, N_{\mathrm{B}}: k+b+i M_{\mathrm{T}} \in \mathcal{P}\right\}$. The power of each beam is distributed uniformly among its resources, i.e., $\gamma_{k}[p, b]=1 /\left|\mathcal{R}_{k}\right|$. The noise variance is $\sigma_{\eta}^{2}=10^{0.1\left(n_{\mathrm{Rx}}+N_{0}\right)} N \Delta f$, where $N_{0}=-174 \mathrm{dBm} \mathrm{Hz}^{-1}$ is the noise power spectral density per dimension and $n_{\mathrm{Rx}}=8 \mathrm{~dB}$ is the Rx noise figure. The standard deviation of the clock offset is $\sigma_{\text {clk }}=2 /(N \Delta f)$, so that $c \sigma_{\mathrm{clk}} \approx 4.88 \mathrm{~m}$. We use a DFT beam codebook:

$$
\boldsymbol{f}_{k}=\left[1, \mathrm{e}^{-\mathrm{j} \frac{2 \pi}{N_{\mathrm{T}}} k}, \ldots, \mathrm{e}^{-\mathrm{j} \frac{2 \pi}{N_{\mathrm{T}}}\left(N_{\mathrm{T}}-1\right) k}\right], k=1, \ldots, M_{\mathrm{T}}=N_{\mathrm{T}}
$$

3) Benchmark for beam power allocation: In order to fairly evaluate our power allocation strategies, we set as benchmark the uniform power allocation to beams exciting useful directions. For a given confidence level $\kappa$ we get a grid of AODs for each path as in (38) and compute the set of useful beams as

$$
\mathcal{B}_{\mathrm{uni}}^{(\kappa)}=\cup_{l=0}^{L-1} \cup_{m=0}^{N_{\theta}}\left\{\underset{k=1, \ldots, N_{\mathrm{T}}}{\operatorname{argmax}}\left|\boldsymbol{a}_{\mathrm{T}}^{\mathrm{T}}\left(\theta_{\mathrm{T}, l, m}^{(\kappa)}\right) \boldsymbol{f}_{k}\right|\right\} .
$$

The power allocation vector $\boldsymbol{q}$ is

$$
q_{k}= \begin{cases}1 /\left|\mathcal{B}_{\text {uni }}^{(\kappa)}\right|, & k \in \mathcal{B}_{\text {uni }}^{(\kappa)} \\ 0, & k \notin \mathcal{B}_{\text {uni }}^{(\kappa)}\end{cases}
$$

\section{B. Power allocation strategies and position estimation algorithm parameters}

The power allocation strategies and their corresponding parametrizations that we consider for our simulation results are as follows:

- opt. unconstr: Solution of (37). The number of points of known cubatures of 5th degree (in order to ensure a sufficiently dense sampling of the support of the distribution) with positive weights is $2^{11}+2 \cdot 11=4118$, which incurs prohibitive computational complexity. Instead, we draw $11^{2}=121$ random samples (as many as the lower bound for any cubature) from the joint 11-dimensional distribution.

- opt. constr: Solution of (37) with 121 random samples from the joint 11-dimensional distribution and additional constraints (40), with $\kappa=0.995, q_{\mathrm{th}}=-10 \mathrm{~dB}$ and $N_{\theta}=15$. 


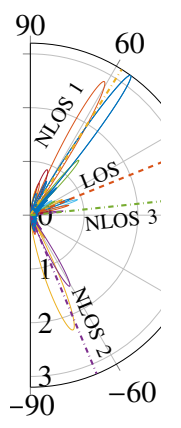

(a) opt. unconstr.

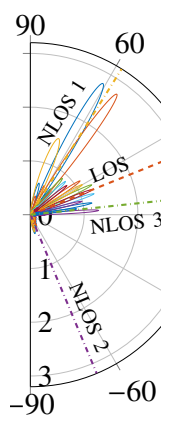

(d) subopt.

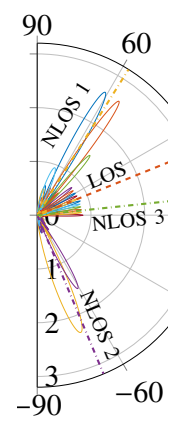

(b) opt. constr.

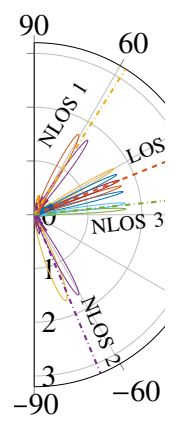

(e) uni 0.60

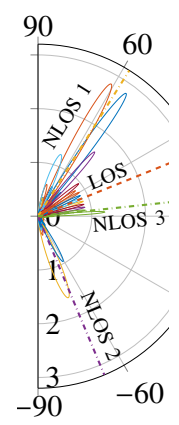

(c) opt. reduced

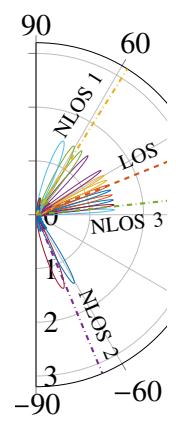

(f) uni 0.90

Fig. 3. Beam patterns $\left|\boldsymbol{a}_{\mathrm{T}}^{\mathrm{T}}\left(\theta_{\mathrm{T}}\right) \boldsymbol{f}_{k} \sqrt{q_{k}}\right|, k=1, \ldots, M_{\mathrm{T}}$, for different power allocation strategies.

- opt. reduced: Solution of the minimization of the lower bound on ESPEB (42) with $8^{2}=64$ random samples from the joint 8-dimensional distribution and additional constraints (40), with $\kappa=0.995, q_{\text {th }}=-10 \mathrm{~dB}$ and $N_{\theta}=15$.

- subopt.: Solution of 43)-45, with 9-point cubatures for the involved 2D marginals, $\kappa=$ $0.995, q_{\text {th,LOS }}=-3 \mathrm{~dB}, q_{\mathrm{th}}=-10 \mathrm{~dB}$ and $N_{\theta}=15$.

- uni $\kappa$ : Uniform power allocation to useful directions, according to (66)- 67), with $\kappa=$ $\{0.60,0.90\}$ and $N_{\theta}=15$. We note that choosing $\kappa=0.995$ as for the other strategies results in performance degradation; hence, results for this value are not icluded.

The beampatterns of the power allocation strategies for the considered prior knowledge are shown in Fig. 3. We observe in Figs. 3(a)-(d) that for the optimized power allocation strategies, most of the available power is spent on beams illuminating NLOS paths. When $\sigma_{\text {clk }}$ is very small (i.e. when the synchronization error is very small), having only the delay measurement of the LOS suffices to determine the distance between the base station (BS) and the user equipment (UE). However, as $\sigma_{\text {clk }}$ increases, neither the LOS nor the NLOS provide individually information about 
the BS-UE distance. In these cases, they are the differences between delays that are informative, and this implies that several paths (not only one) have to be illuminated with sufficient power because if there is a large power unbalance between rays, then the delay differences will not be estimated precisely. Comparing Fig. 3.a) with Figs. 3.(b)-(d), we see that when the constraints (40) are not applied, the power allocation to NLOS components is more significant, with the power invested to less likely LOS directions being very low. From Figs. 3(b) and (c), we can see that the impact of the dimensionality reduction (42) is the reduction of the power spent on the 2nd NLOS path. This is explained by the fact that the fading of the path gains is not taken into account; hence, for the mean values of the path gains, more power is spent on the paths that offer more useful position information. Also, in Fig. 3(d) we observe that our suboptimal approach allocates almost no power to the 2nd NLOS path, as in the last step where all paths are considered jointly, only the receiver's location uncertainty and the mean scatterers'/reflectors' locations are taken into account; for this setup, the information offered by the 1st NLOS path is more useful and therefore most of the available power is allocated for its illumination. For the uniform allocation, higher confidence values lead to activation of more beams and spreading of the avaiable power to more directions.

Regarding the position estimation algorithm parameters, we set $N_{\tau}=2 N_{\mathrm{P}}, N_{\theta_{\mathrm{T}}}=2 N_{\mathrm{T}}, N_{\theta_{\mathrm{R}}}=$ $2 N_{\mathrm{R}}, P_{\mathrm{fa}}=0.05, \zeta_{1}$ is pre-trained for the given $P_{\mathrm{fa}}$ and power allocation strategy, $\zeta_{2}=-35 \mathrm{~dB}$, $N_{\mathrm{cd}}=3, L_{\max }=10, \chi=\sigma_{\eta} \sqrt{2\left(N_{\mathrm{T}}+N_{\mathrm{R}}\right)|\mathcal{P}| N_{\mathrm{B}} P_{\mathrm{RE}} / N_{\mathrm{T}}}$ (chosen according to [51]), $\zeta_{3, a}=10^{-4}$ and $\zeta_{3, b}=10^{-2}$.

\section{Performance vs SNR for fixed geometry}

We fix the geometry and the reflection coefficients to their mean value $\boldsymbol{\mu}$ in (63) to examine the performance of the position estimation algorithm as a function of the Tx power. For the power allocation strategies described in Sec. VI-B, in Fig. 4, we plot the position root mean square error (RMSE) $\mathbb{E}_{\boldsymbol{\eta}, \epsilon_{\mathrm{clk}}}\left[\left\|\hat{\boldsymbol{p}}_{\mathrm{R}}-\boldsymbol{p}_{\mathrm{R}}\right\|_{2}^{2}\right]$ and PEB as functions of the average power per resource element $P_{\mathrm{RE}}=P_{\text {tot }} /\left(N_{\mathrm{B}} N_{\mathrm{P}}\right)$, with $\hat{p}_{\mathrm{R}}$ being the position estimate. We note that the average Tx power $P_{\mathrm{T}}$ is related to $P_{\mathrm{RE}}$ as $P_{\mathrm{T}}=P_{\mathrm{RE}} N_{\mathrm{P}} / N$.

We can see that the bound is attained for all power allocation strategies. Regarding uniform power allocation, the distance of the RMSE from the bound for low Tx power is attributed to the fact that, although the LOS path is detected, the probability of detection for the NLOS is small. With only the LOS path being detected, the clock offset cannot be resolved and the 


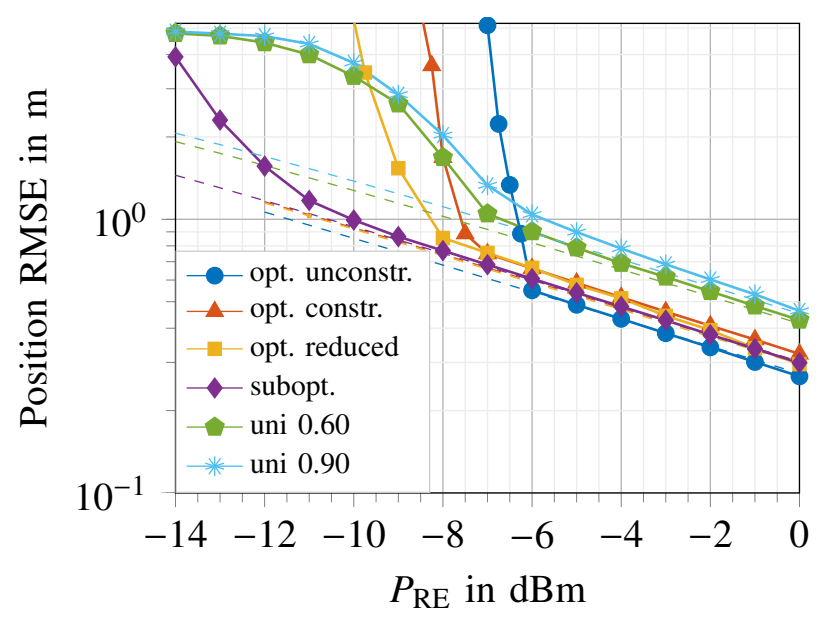

Fig. 4. Position RMSE (solid lines) and PEB (dashed lines) vs Tx power for different power allocation strategies.

resulting position RMSE approaches the standard deviation of the clock offset $c \cdot \sigma_{\mathrm{clk}} \approx 4.88 \mathrm{~m}$. Among the two considered configurations $(\kappa=0.60$ and $\kappa=0.90)$, the former has slightly better performance, as the available power is more concentrated to the true location of the $\mathrm{Rx}$ and the reflectors. But, as we will see later on, this comes with a cost, when the uncertainty about the geometry is considered.

The optimized allocation strategies result in similar PEBs and offer significant improvement compared to the uniform, with a gain of 3 to $4 \mathrm{~dB}$ for the same localization accuracy. The lowest PEB is attained by "opt. unconstr.", but the RMSE converges to the PEB for larger $P_{\mathrm{RE}}$, compared to the other strategies. The reason for this behavior is that, as can be observed in Fig. 3. a), only a small fraction of power is spent in the LOS direction and the Tx power required for the LOS path to be detected is larger. When the LOS path is missed, the first arriving NLOS path is treated as LOS by the algorithm, resulting in a large position error. Due to the constraints (40), the rest of the proposed strategies ("opt. constr.", "opt. reduced" and "subopt.") allocate more power to the LOS, enabling to attain the PEB at lower values of $P_{\mathrm{RE}}$, with only a small performance penatly. The RMSE of "opt. reduced" converges slightly faster to the bound compared to "opt. constr.", as slightly more power is allocated to the LOS path. The "subopt." allocation exhibits the most robust performance, as the LOS path can be detected for much lower Tx power values. 


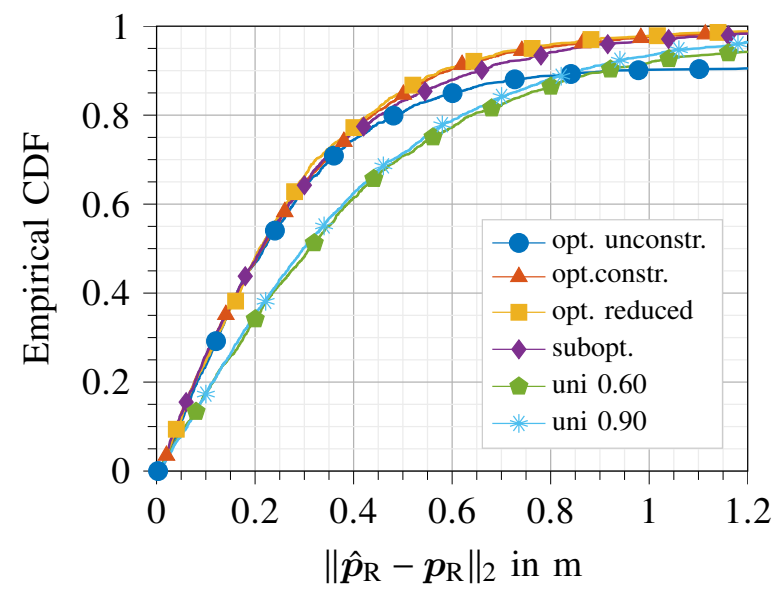

Fig. 5. Empirical cdf of $\left\|\hat{p}_{\mathrm{R}}-\boldsymbol{p}_{\mathrm{R}}\right\|_{2}$ for different power allocation strategies.

TABLE I

PERCENTILES OF THE CDF OF THE POSITION ERROR IN M FOR DIFFERENT POWER ALLOCATION STRATEGIES.

\begin{tabular}{r|cccc} 
& $50 \%$ & $90 \%$ & $95 \%$ & $99 \%$ \\
\hline \hline opt. unconstr. & 0.22 & 0.93 & 29.55 & 72.25 \\
opt. constr. & 0.21 & 0.59 & 0.78 & 1.32 \\
opt. reduced & 0.21 & 0.57 & 0.76 & 1.25 \\
subopt. & 0.21 & 0.65 & 0.84 & 1.45 \\
uni 0.60 & 0.31 & 0.91 & 1.30 & 20.83 \\
uni 0.90 & 0.30 & 0.86 & 1.10 & 1.96
\end{tabular}

\section{Performance with random geometry}

The results in Fig. 4 and the corresponding discussion were useful in examining the behavior of the position estimation algorithm, but do not provide a complete characterization of the performance of the power allocation strategies. To better evaluate their performance, for $P_{\mathrm{RE}}=$ $0 \mathrm{dBm}$ and the rest of the system parameters as described in Sec. VI-A2, we plot in Fig. 5 the cumulative distribution function (cdf) of the position error $\left\|\hat{p_{R}}-p_{R}\right\|_{2}$, which is computed drawing samples from (63)-(64). A summary of the percentiles of the distribution of the position error is provided in Table I.

We can observe in Fig. 5 and Table 10 that "opt. reduced" and "opt. constr." achieve the best performance. The latter is slightly worse at higher percentiles, as more points would be required for a more accurate approximation of the expectation in the corresponding optimization problem. 
In spite of the lower computations cost of the "subopt." allocation, its performance degradation is almost unnoticeable. On the other hand, the "opt. unconstr." approach, although attaining almost the same median error as the other optimized strategies, has much lower accuracy for higher percentiles. This is attributed to the low power spent in the direction around the LOS path, resulting in low probability of detection of the LOS. Compared to the best of the uniform allocations, the "opt. reduced" power allocation offers a position error reduction of 30\%, 34\%, $31 \%$ and $36 \%$ at the $50 \%, 90 \%, 95 \%$ and $99 \%$ percentile, respectively.

Regarding the uniform allocations, we can see that spreading the power to a reduced set of beams ("uni 0.60") might result in better positioning accuracy for some geometry realizations, as seen for example in Fig. 4, but it significantly deteriorates the performance for other possible realizations. This explains the higher values of position errors at the upper percentiles of the corresponding cdf.

\section{E. Power allocation as a function of $\sigma_{c l k}$}

We now examine the effect of $\sigma_{\text {clk }}$ on the power allocation. First, similar to 66, we define the set of LOS-illuminating beams as

$$
\mathcal{B}_{\mathrm{LOS}}^{(\kappa)}=\cup_{m=0}^{N_{\theta}}\left\{\underset{k=1, \ldots, N_{\mathrm{T}}}{\operatorname{argmax}}\left|\boldsymbol{a}_{\mathrm{T}}^{\mathrm{T}}\left(\theta_{\mathrm{T}, 0, m}^{(\kappa)}\right) \boldsymbol{f}_{k}\right|\right\} .
$$

and the fraction of power spent on them as

$$
q_{\mathrm{LOS}}=\sum_{k \in \mathcal{B}_{\mathrm{LOS}}^{(\kappa)}} q_{k} .
$$

In Fig. 6(a) we plot $q_{\text {LOS }}$ as a function of $\sigma_{\text {clk }}$ for the power allocation strategies "opt. unconstr.", "opt. constr.", "subopt" and "uni 0.90", for $N_{\mathrm{R}}=\{4,16\}, P_{\mathrm{RE}}=0 \mathrm{dBm}, \kappa=0.995$ and the rest

of the system parameters as described in Sec. VI-A2, in Fig. 6.b) we plot the corresponding $\mathbb{E}[\mathrm{PEB}]$. We can see in Fig. 6(a) that for very low values of $\sigma_{\mathrm{clk}}$, equivalent to almost perfect Tx-Rx synchronization, it is optimal to spend almost all the available power on LOS-illuminating beams. As $\sigma_{\mathrm{clk}}$ increases, $q_{\mathrm{LOS}}$ decreases rapidly for both optimized allocation strategies, until it saturates at a relatively low value. This is explained as follows: The clock offset decreases the amount of range information provided by the LOS path and the larger standard deviation of the clock offset, the more significant the decrease. Hence, as $\sigma_{\text {clk }}$ increases, the ranging information provided by the NLOS paths becomes more significant and, therefore, more power is spent on them. Nevertheless, the saturation occurs because the measurement of the LOS AOD offers 


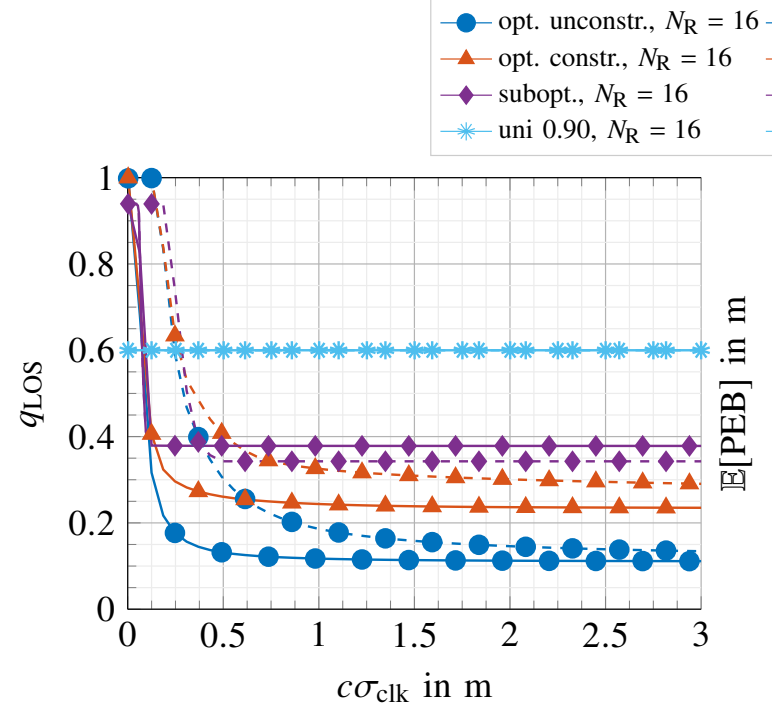

(a) $q_{\text {LOS }}$ vs. $\sigma_{\text {clk }}$
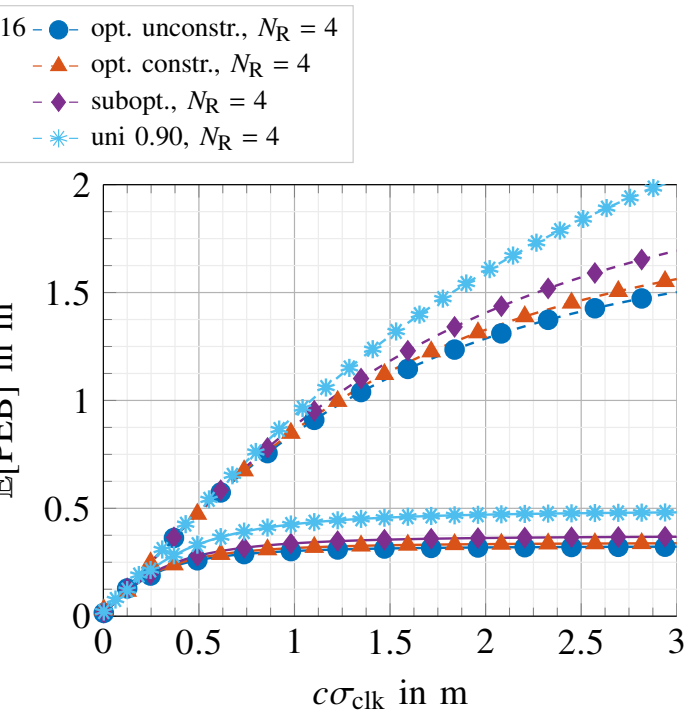

(b) $\mathbb{E}[\mathrm{PEB}]$ vs. $\sigma_{\mathrm{clk}}$

Fig. 6. Fraction of power allocated to LOS-illuminating beams $q_{\mathrm{LOS}}$ and $\mathbb{E}[\mathrm{PEB}]$ as functions of $\sigma_{\text {clk }}$.

significant information in the orthogonal direction, which is reduced when $q_{\text {LOS }}$ is decreased. The saturation value for "opt. constr." is higher due to the additional constraints on LOS illumination. Also, we observe that the transition from high to low $q_{\text {LOS }}$ values is slower for $N_{\mathrm{R}}=4$. This is attributed to the fact that NLOS paths offer rank-1 position information, whose intensity depends on the quality of the TOA, AOD and AOA measurements combined [12]. For $N_{\mathrm{R}}=4$, the quality of the AOA measurement is lower; therefore, the intensity of the ranging information from the NLOS paths is smaller, compared to $N_{\mathrm{R}}=16$, and becomes significant for larger values of $\sigma_{\text {clk }}$.

In Fig. 6(b) we see that $\mathbb{E}[\mathrm{PEB}]$ increases with increasing $\sigma_{\mathrm{clk}}$, until it saturates at a value dependent on the power allocation strategy and the system configuration $\left(N_{\mathrm{R}}=\{4,16\}\right)$. As $\sigma_{\text {clk }}$ increases the reduction of ranging information from the LOS path cannot be complemented by ranging information from the NLOS paths (even with optimized power allocation), resulting in a larger error. In the saturation region the ranging information from the LOS path becomes negligible compared to the clock offset-independent part of ranging information offered by the combination of NLOS paths with the LOS path.

\section{CONCLUSION}

Optimal power allocation on a beam codebook for single-anchor localization and lowercomplexity suboptimal alternatives have been considered under imperfect Tx-Rx synchronization. 
A channel and position estimation method has also been proposed. Numerical results show that our suboptimal power allocation approach offers a good balance between performance and complexity, as the significant complexity reduction for the computation of the power allocation incurs only a very small performance penalty. Our analysis has shown that even for low values of the clock offset standard deviation it is optimal according to the CRLB to allocate most of the available power to scatterer/reflector illuminating beams to recover necessary range information. We have also shown that guaranteeing a minimum amount of power spent on LOS-illuminating beams, can be beneficial when the actual position estimation is considered, as it ensures that LOS path is detected with a high probability. The proposed position estimation algorithm attains the corresponding CRLB for all considered power allocation strategies, benefiting from the gridless parameter estimation, which avoids the appearance of spurious paths due to grid mismatch, while also filtering out noisy detected paths exploiting the information on the clock offset carried by single-bounce-NLOS paths.

\section{APPENDIX}

\section{POWER AlLOCATION FOR THE LOS PATH}

Here we show how to formulate (43) as an SDP using only a 1D quadrature rule for the approximation of the expectation over $\theta_{\mathrm{T}, 0}$. This is accomplished in two steps:

- In the first step we show that the integration over $d_{0}$ and $\theta_{\mathrm{T}, 0}$ can be carried out separately;

- in the second step, after averaging over $d_{0}$, we exploit the form of the resulting function of $\theta_{\mathrm{T}, 0}$ and formulate the problem as an SDP.

We write $\mathbb{E}_{d_{0}, \theta_{\mathrm{T}, 0}}[\cdot]$ instead of $\mathbb{E}_{\boldsymbol{p}_{\mathrm{R}}}[\cdot]$. Also, for notational brevity we write

$$
\overline{\boldsymbol{J}}=\mathbb{E}_{\alpha_{\mathrm{R}}, \boldsymbol{h}_{0} \mid d_{0}, \theta_{\mathrm{T}, 0}}\left[\boldsymbol{J}_{\boldsymbol{\nu}_{\mathrm{LOS}}}\left(\boldsymbol{q}, d_{0}, \theta_{\mathrm{T}, 0}, \alpha_{\mathrm{R}}, \boldsymbol{h}_{0}\right)\right] .
$$

We index the elements of $\overline{\boldsymbol{J}}$ with the pair of parameters to which they correspond.

First, after some algebra we find that

where

$$
\operatorname{tr}\left(\boldsymbol{E}^{\mathrm{T}} \overline{\boldsymbol{J}}^{-1} \boldsymbol{E}\right)=\frac{c^{2}}{\bar{J}_{\tau_{0}, \tau_{0}}-\frac{\bar{J}_{\tau_{0}, \theta_{\mathrm{T}, 0}}^{2}}{\bar{J}_{\theta_{\mathrm{T}, 0}, \theta_{\mathrm{T}, 0}}}}+\frac{d_{0}^{2}}{\bar{J}_{\theta_{\mathrm{T}, 0}, \theta_{\mathrm{T}, 0}}-\frac{\bar{J}_{\tau_{0}, \theta_{\mathrm{T}, 0}}^{2}}{\bar{J}_{\tau_{0}, \tau_{0}}}}+c^{2} \sigma_{\mathrm{clk}}^{2},
$$

$$
\begin{aligned}
& \bar{J}_{a, b}=\mathbb{E}_{\alpha_{\mathrm{R}}, h_{0} \mid d_{0}, \theta_{\mathrm{T}, 0}}\left[J_{a, b}\right] \\
& J_{a, b}=\frac{2}{\sigma_{\eta}^{2}} \sum_{b=1}^{N_{\mathrm{B}}} \sum_{p \in \mathcal{P}} \mathfrak{R}\left\{\frac{\partial \boldsymbol{m}_{b}^{\mathrm{H}}[p]}{\partial a} \frac{\partial \boldsymbol{m}_{b}[p]}{\partial b}\right\}
\end{aligned}
$$


with $a, b \in\left\{d_{0}, \theta_{\mathrm{T}, 0}\right\}$. We can show that $J_{a, b}, a, b \in\left\{d_{0}, \theta_{\mathrm{T}, 0}\right\}$, are independent of $\alpha_{\mathrm{R}}$ and the phase of $h_{0}$. Hence, they can be expressed as

$\bar{J}_{a, b}=\mathbb{E}_{\boldsymbol{h}_{0} \mid d_{0}, \theta_{\mathrm{T}, 0}}\left[J_{a, b}\left(\boldsymbol{q}, \theta_{\mathrm{T}, 0},\left|h_{0}\left(d_{0}\right)\right|^{2}\right)\right]=\mathbb{E}_{\boldsymbol{h}_{0} \mid d_{0}, \theta_{\mathrm{T}, 0}}\left[\left|h_{0}\left(d_{0}\right)\right|^{2} j_{a, b}\left(\boldsymbol{q}, \theta_{\mathrm{T}, 0}\right)\right]=g_{0}\left(d_{0}\right) j_{a, b}\left(\boldsymbol{q}, \theta_{\mathrm{T}, 0}\right)$,

where $g_{0}\left(d_{0}\right)=\mathbb{E}_{\boldsymbol{h}_{0} \mid d_{0}}\left[\left|h_{0}\left(d_{0}\right)\right|^{2}\right]$ and $j_{a, b}\left(\boldsymbol{q}, \theta_{\mathrm{T}, 0}\right)=J_{a, b}\left(\boldsymbol{q}, \theta_{\mathrm{T}, 0},\left|h_{0}\left(d_{0}\right)\right|^{2}\right) /\left|h_{0}\left(d_{0}\right)\right|^{2}$ is a function of $\boldsymbol{q}$ and $\theta_{\mathrm{T}, 0}$. For the second equality in (74), we used the fact that $J_{a, b}$ can be expressed as the product of two terms, one dependent on the gain magnitude and the other on $\boldsymbol{q}$ and $\theta_{\mathrm{T}, 0}$. We can then rewrite (71) as

$$
\operatorname{tr}\left(\boldsymbol{E}^{\mathrm{T}} \overline{\boldsymbol{J}}^{-1} \boldsymbol{E}\right)=\frac{1}{g_{0}\left(d_{0}\right)}\left(\frac{c^{2}}{I_{\tau_{0}}\left(\boldsymbol{q}, \theta_{\mathrm{T}, 0}\right)}+\frac{d_{0}^{2}}{I_{\theta_{\mathrm{T}, 0}}\left(\boldsymbol{q}, \theta_{\mathrm{T}, 0}\right)}\right)+c^{2} \sigma_{\mathrm{clk}}^{2},
$$

where

$$
\begin{gathered}
I_{\tau_{0}}\left(\boldsymbol{q}, \theta_{\mathrm{T}, 0}\right)=j_{\tau_{0}, \tau_{0}}\left(\boldsymbol{q}, \theta_{\mathrm{T}, 0}\right)-\frac{j_{\tau_{0}, \theta_{\mathrm{T}, 0}}^{2}\left(\boldsymbol{q}, \theta_{\mathrm{T}, 0}\right)}{j_{\theta_{\mathrm{T}, 0}, \theta_{\mathrm{T}, 0}}\left(\boldsymbol{q}, \theta_{\mathrm{T}, 0}\right)} \\
I_{\theta_{\mathrm{T}, 0}}\left(\boldsymbol{q}, \theta_{\mathrm{T}, 0}\right)=j_{\theta_{\mathrm{T}, 0}, \theta_{\mathrm{T}, 0}}\left(\boldsymbol{q}, \theta_{\mathrm{T}, 0}\right)-\frac{j_{\tau_{0}, \theta_{\mathrm{T}, 0}}^{2}\left(\boldsymbol{q}, \theta_{\mathrm{T}, 0}\right)}{j_{\tau_{0}, \tau_{0}}\left(\boldsymbol{q}, \theta_{\mathrm{T}, 0}\right)} .
\end{gathered}
$$

It is then apparent from the form of the function in (75) that integration over $d_{0}$ and $\theta_{\mathrm{T}, 0}$ can be carried out separately.

For the second step, taking the expectation over $d_{0}$ and defining

$$
\begin{aligned}
& \bar{g}_{0}\left(\theta_{\mathrm{T}, 0}\right)=1 / \mathbb{E}_{d_{0} \mid \theta_{\mathrm{T}, 0}}\left[1 / g_{0}\left(d_{0}\right)\right] \\
& \bar{d}_{0}\left(\theta_{\mathrm{T}, 0}\right)=\sqrt{\mathbb{E}_{d_{0} \mid \theta_{\mathrm{T}, 0}}\left[\frac{\bar{g}_{0}\left(\theta_{\mathrm{T}, 0}\right)}{g_{0}\left(d_{0}\right)} d_{0}^{2}\right]}
\end{aligned}
$$

we get

$$
\mathbb{E}_{d_{0} \mid \theta_{\mathrm{T}, 0}}\left[\operatorname{tr}\left(\boldsymbol{E}^{\mathrm{T}} \overline{\boldsymbol{J}}^{-1} \boldsymbol{E}\right)\right]=\frac{1}{\bar{g}_{0}\left(\theta_{\mathrm{T}, 0}\right)}\left(\frac{c^{2}}{I_{\tau_{0}}\left(\boldsymbol{q}, \theta_{\mathrm{T}, 0}\right)}+\frac{\left(\bar{d}_{0}\left(\theta_{\mathrm{T}, 0}\right)\right)^{2}}{I_{\theta_{\mathrm{T}, 0}}\left(\boldsymbol{q}, \theta_{\mathrm{T}, 0}\right)}\right)+c^{2} \sigma_{\mathrm{clk}}^{2} .
$$

Comparing (80) to (71), we can conclude that, in order to be able to formulate the problem in a convex form, $\mathbb{E}_{d_{0} \mid \theta_{\mathrm{T}, 0}}\left[\operatorname{tr}\left(\boldsymbol{E}^{\mathrm{T}} \overline{\boldsymbol{J}}^{-1} \boldsymbol{E}\right)\right]$ can be expressed as

$$
\mathbb{E}_{d_{0} \mid \theta_{\mathrm{T}, 0}}\left[\operatorname{tr}\left(\boldsymbol{E}^{\mathrm{T}} \check{\boldsymbol{J}}^{-1} \boldsymbol{E}\right)\right]=\operatorname{tr}\left(\boldsymbol{E}^{\mathrm{T}} \boldsymbol{J}_{\nu_{\mathrm{L} O S}}^{-1}\left(\boldsymbol{q}, \bar{d}_{0}\left(\theta_{\mathrm{T}, 0}\right), \theta_{\mathrm{T}, 0}, \check{\alpha}_{\mathrm{R}}, \sqrt{\bar{g}_{0}\left(\theta_{\mathrm{T}, 0}\right)} \mathrm{e}^{\mathrm{j} \beta_{g}}\right) \boldsymbol{E}\right)
$$

where $\check{\alpha}_{\mathrm{R}}$ and $\beta_{g}$ can be chosen arbitrarily, since they do not have an impact on the objective. Finally, using (81) and the identity

$$
\mathbb{E}_{d_{0}, \theta_{\mathrm{T}, 0}}\left[\operatorname{tr}\left(\boldsymbol{E}^{\mathrm{T}} \overline{\boldsymbol{J}} \boldsymbol{E}\right)\right]=\mathbb{E}_{\theta_{\mathrm{T}, 0}}\left[\mathbb{E}_{d_{0} \mid \theta_{\mathrm{T}, 0}}\left[\operatorname{tr}\left(\boldsymbol{E}^{\mathrm{T}} \overline{\boldsymbol{J}}^{-1} \boldsymbol{E}\right)\right]\right],
$$


we can employ a $1 \mathrm{D}$ quadrature rule to approximate the expectation integral over $\theta_{\mathrm{T}, 0}$ to get the following SDP:

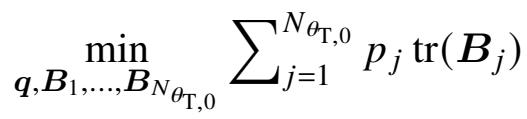

$$
\begin{aligned}
& \text { s.t. } \boldsymbol{q} \geq \mathbf{0}, \mathbf{1}^{\mathrm{T}} \boldsymbol{q} \leq 1, \\
& {\left[\begin{array}{cc}
\boldsymbol{B}_{j} & \boldsymbol{E}^{\mathrm{T}} \\
\boldsymbol{E} & \boldsymbol{J}_{\boldsymbol{\nu}_{\mathrm{LOS}}}\left(\boldsymbol{q}, \bar{d}_{0}\left(\theta_{\mathrm{T}, 0, j}\right), \theta_{\mathrm{T}, 0, j}, \check{\alpha}_{\mathrm{R}}, \sqrt{\bar{g}_{0}\left(\theta_{\mathrm{T}, 0, j}\right)} \mathrm{e}^{\left.\mathrm{j} \beta_{g}\right)}\right.
\end{array}\right] \geq \mathbf{0 ,} j=1, \ldots, N_{\theta_{\mathrm{T}, 0}} .}
\end{aligned}
$$

\section{REFERENCES}

[1] S. Mumtaz, J. Rodriguez, and L. Dai, mmWave Massive MIMO: A Paradigm for 5G. Academic Press, 2017.

[2] A. L. Swindlehurst, E. Ayanoglu, P. Heydari, and F. Capolino, "Millimeter-wave massive MIMO: the next wireless revolution?” IEEE Commun. Mag., vol. 52, no. 9, pp. 56-62, Sep. 2014.

[3] F. Wen, H. Wymeersch, B. Peng, W. P. Tay, H. C. So, and D. Yang, "A survey on 5G massive MIMO localization,” Digital Signal Processing, vol. 94, pp. 21 - 28, Nov. 2019, Special Issue on Source Localization in Massive MIMO.

[4] J. A. del Peral-Rosado, R. Raulefs, J. A. López-Salcedo, and G. Seco-Granados, "Survey of cellular mobile radio localization methods: From 1G to 5G," IEEE Commun. Surveys Tuts., vol. 20, no. 2, pp. 1124-1148, 2nd quarter 2018.

[5] R. Keating, M. Säily, J. Hulkkonen, and J. Karjalainen, "Overview of positioning in 5G new radio," in Proc. 16th Int. Symposium on Wireless Commun. Systems (ISWCS), Aug. 2019, pp. 320-324.

[6] 3rd Generation Partnership Project (3GPP), "Technical Specification Group Services and System Aspects; Study on positioning use cases; Stage 1 (Release 16),” TR22.872 V16.1.0, Aug. 2019.

[7] H. Wymeersch, G. Seco-Granados, G. Destino, D. Dardari, and F. Tufvesson, "5G mmWave positioning for vehicular networks," IEEE Wireless Commun., vol. 24, no. 6, pp. 80-86, Dec. 2017.

[8] A. Shahmansoori, G. E. Garcia, G. Destino, G. Seco-Granados, and H. Wymeersch, "5G position and orientation estimation through millimeter wave MIMO,” in Proc. IEEE GlOEBCOM Workshops (GC Wkshps), San Diego, CA, Dec. 2015, pp. $1-6$.

[9] Z. Abu-Shaban, X. Zhou, T. D. Abhayapala, G. Seco-Granados, and H. Wymeersch, "Error bounds for uplink and downlink 3D localization in 5G mmWave systems," IEEE Trans. Wireless Commun., vol. 17, no. 8, pp. 4939-4954, Aug. 2018.

[10] A. Guerra, F. Guidi, and D. Dardari, "Single-anchor localization and orientation performance limits using massive arrays: MIMO vs. beamforming," IEEE Trans. Wireless Commun., vol. 17, no. 8, pp. 5241-5255, Aug. 2018.

[11] R. Mendrzik, H. Wymeersch, G. Bauch, and Z. Abu-Shaban, "Harnessing NLOS components for position and orientation estimation in 5G millimeter wave MIMO,” IEEE Trans. Wireless Commun., vol. 18, no. 1, pp. 93-107, Jan. 2019.

[12] A. Kakkavas, M. H. Castañeda García, R. A. Stirling-Gallacher, and J. A. Nossek, "Performance limits of single-anchor millimeter-wave positioning," IEEE Trans. Wireless Commun., vol. 18, no. 11, pp. 5196-5210, Nov. 2019.

[13] A. Shahmansoori, G. E. Garcia, G. Destino, G. Seco-Granados, and H. Wymeersch, "Position and orientation estimation through millimeter-wave MIMO in 5G systems," IEEE Trans. Wireless Commun., vol. 17, no. 3, pp. 1822-1835, Mar. 2018.

[14] J. Talvitie, M. Koivisto, T. Levanen, M. Valkama, G. Destino, and H. Wymeersch, "High-accuracy joint position and orientation estimation in sparse 5G mmWave channel," in Proc. IEEE Int. Conf. Commun. (ICC), Shanghai, China, May 2019, pp. 1-7. 
[15] J. Palacios, G. Bielsa, P. Casari, and J. Widmer, "Single- and multiple-access point indoor localization for millimeter-wave networks," IEEE Trans. Wireless Commun., vol. 18, no. 3, pp. 1927-1942, Mar. 2019.

[16] Y. Liu, Y. Shen, and M. Z. Win, "Single-anchor localization and synchronization of full-duplex agents," IEEE Trans. Commun., vol. 67, no. 3, pp. 2355-2367, Mar. 2019.

[17] T. Wang, H. Zhao, and Y. Shen, "An efficient single-anchor localization method using ultra-wide bandwidth systems," Applied Sciences, vol. 10, no. 1, Dec. 2019.

[18] M. Rath, J. Kulmer, E. Leitinger, and K. Witrisal, "Single-anchor positioning: Multipath processing with non-coherent directional measurements," IEEE Access, vol. 8, pp. 88 115-88 132, May 2020.

[19] A. Fascista, A. Coluccia, H. Wymeersch, and G. Seco-Granados, "Millimeter-wave downlink positioning with a singleantenna receiver," IEEE Trans. Wireless Commun., vol. 18, no. 9, pp. 4479-4490, Sep. 2019.

[20] — "Low-complexity accurate mmWave positioning for single-antenna users based on angle-of-departure and adaptive beamforming," in Proc. IEEE Int. Conf. Acoustics, Speech and Signal Process. (ICASSP), Barcelon, Spain, May 2020, pp. 4866-4870.

[21] C. Gentner, T. Jost, W. Wang, S. Zhang, A. Dammann, and U. Fiebig, "Multipath assisted positioning with simultaneous localization and mapping," IEEE Trans. Wireless Commun., vol. 15, no. 9, pp. 6104-6117, Sep. 2016.

[22] E. Leitinger, F. Meyer, F. Hlawatsch, K. Witrisal, F. Tufvesson, and M. Z. Win, "A belief propagation algorithm for multipath-based SLAM," IEEE Trans. Wireless Commun., vol. 18, no. 12, pp. 5613-5629, Dec. 2019.

[23] H. Wymeersch, N. Garcia, H. Kim, G. Seco-Granados, S. Kim, F. Wen, and M. Fröhle, "5G mmWave downlink vehicular positioning," in Proc. IEEE Global Commun. Conf. (GLOBECOM), Abu Dhabi, UAE, Dec. 2018, pp. $206-212$.

[24] J. Talvitie, T. Levanen, M. Koivisto, K. Pajukoski, M. Renfors, and M. Valkama, "Positioning of high-speed trains using 5G new radio synchronization signals," in Proc. IEEE Wireless Commun. and Netw. Conf. (WCNC), Apr. 2018, pp. 1-6.

[25] X. Li, E. Leitinger, M. Oskarsson, K. Åström, and F. Tufvesson, "Massive MIMO-based localization and mapping exploiting phase information of multipath components," IEEE Trans. Wireless Commun., vol. 18, no. 9, pp. 4254-4267, Sep. 2019.

[26] R. Mendrzik, F. Meyer, G. Bauch, and M. Z. Win, "Enabling situational awareness in millimeter wave massive MIMO systems,” IEEE J. Sel. Areas Commun., vol. 13, no. 5, pp. 1196-1211, Sep. 2019.

[27] H. Kim, K. Granström, S. Kim, and H. Wymeersch, "Low-complexity 5G SLAM with CKF-PHD filter," in Proc. IEEE Int. Conf. Acoustics, Speech and Signal Process. (ICASSP), Barcelona, Spain, May 2020, pp. 5220-5224.

[28] B. Zhou, A. Liu, and V. Lau, "Successive localization and beamforming in 5G mmWave MIMO communication systems," IEEE Trans. Signal Process., vol. 67, no. 6, pp. 1620-1635, Mar. 2019.

[29] N. Garcia, H. Wymeersch, and D. T. M. Slock, "Optimal precoders for tracking the AoD and AoA of a mmWave path," IEEE Trans. Signal Process., vol. 66, no. 21, pp. 5718-5729, Nov. 2018.

[30] R. Koirala, B. Denis, D. Dardari, and B. Uguen, "Localization bound based beamforming optimization for multicarrier mmWave MIMO," in 14th Workshop on Positioning, Navigation and Communications (WPNC), Bremen, Germany, Oct. 2017, pp. 1-6.

[31] R. Koirala, B. Denis, B. Uguen, D. Dardari, and H. Wymeersch, "Localization optimal multi-user beamforming with multicarrier mmwave mimo," in IEEE 29th Annual Int. Symposium Personal, Indoor and Mobile Radio Commun. (PIMRC), Bologna, Italy, Sep. 2018, pp. 1-7.

[32] H. Zhao, L. Zhang, and Y. Shen, "On the optimal beamspace design for direct localization systems," in Proc. IEEE Int. Conf. Commun. (ICC), Kansas City, MO, USA, May 2018, pp. 1-6.

[33] H. Zhao, N. Zhang, and Y. Shen, "Robust beamspace design for direct localization," in Proc. IEEE Int. Conf. Acoustics, Speech and Signal Process. (ICASSP), Brighton, UK, May 2019, pp. 4360-4364. 
[34] W. W. Li, Y. Shen, Y. J. Zhang, and M. Z. Win, "Robust power allocation for energy-efficient location-aware networks," IEEE/ACM Trans. Netw., vol. 21, no. 6, pp. 1918-1930, Dec. 2013.

[35] A. Shahmansoori, G. Seco-Granados, and H. Wymeersch, "Power allocation for OFDM wireless network localization under expectation and robustness constraints," IEEE Trans. Wireless Commun., vol. 16, no. 3, pp. 2027-2038, Mar. 2017.

[36] A. Kakkavas, G. Seco-Granados, H. Wymeersch, M. H. C. Garcia, R. A. Stirling-Gallacher, and J. A. Nossek, "5G downlink multi-beam signal design for LOS positioning," in Proc. IEEE Global Commun. Conf. (GLOBECOM), Waikoloa, HI, USA, Dec. 2019, pp. 1-6.

[37] R. Vaughan and J. Bach-Anderson, Channels, propagation and antennas for mobile communications, ser. Electromagnetic Waves. Stevenage: The Institution of Engineering and Technology, 2003.

[38] T. S. Rappaport, E. Ben-Dor, J. N. Murdock, and Y. Qiao, "38 Ghz and $60 \mathrm{Ghz}$ angle-dependent propagation for cellular \& peer-to-peer wireless communications," in Proc. IEEE Int. Conf. Commun. (ICC), Ottawa, Canada, Jun. 2012, pp. 4568-4573.

[39] M. T. Martinez-Ingles, D. P. Gaillot, J. Pascual-Garcia, J. M. Molina-Garcia-Pardo, M. Lienard, and J. V. Rodríguez, "Deterministic and experimental indoor mmW channel modeling," IEEE Antennas Wireless Propag. Lett., vol. 13, pp. 1047-1050, 2014.

[40] M. Peter et al., "Measurement campaigns and initial channel models for preferred suitable frequency ranges; Deliverable D2.1,” Mar. 2016. [Online]. Available: https://5g-mmmagic.eu/results/\#deliverables

[41] N. Boyd, G. Schiebinger, and B. Recht, "The alternating descent conditional gradient method for sparse inverse problems," SIAM Journal on Optimization, vol. 27, no. 2, pp. 616-639, Apr. 2017.

[42] A. Dammann, T. Jost, R. Raulefs, M. Walter, and S. Zhang, "Optimizing waveforms for positioning in 5G," in Proc. IEEE 17th Int. Workshop on Signal Process. Advances in Wireless Commun. (SPAWC), Edinburgh, UK, Jul. 2016, pp. 1-5.

[43] M. D. Larsen, G. Seco-Granados, and A. L. Swindlehurst, "Pilot optimization for time-delay and channel estimation in OFDM systems," in Proc. IEEE Int. Conf. Acoustics, Speech and Signal Process. (ICASSP), Prague, Czech Republic, May 2011, pp. 3564-3567.

[44] A. Shahmansoori, G. Seco-Granados, and H. Wymeersch, "Robust power allocation for OFDM wireless network localization," in Proc. IEEE Int. Conf. Commun. Workshop (ICCW), London, UK, Jun. 2015, pp. 718-723.

[45] Y. Rockah and P. Schultheiss, "Array shape calibration using sources in unknown locations - Part I: Far-field sources," IEEE Trans. Acoust., Speech, Signal Process., vol. 35, no. 3, pp. 286-299, Mar. 1987.

[46] H. Messer, "The hybrid Cramér-Rao lower bound - from practice to theory," in Proc. Fourth IEEE Workshop on Sensor Array and Multichannel Process. (SAM), Waltham, MA, USA, Jul. 2006, pp. 304-307.

[47] R. Cools, "An encyclopaedia of cubature formulas," Journal of Complexity, vol. 19, no. 3, pp. 445 - 453, Jun. 2003, Oberwolfach Special Issue.

[48] D. Crouse, "Basic tracking using nonlinear 3D monostatic and bistatic measurements," IEEE Aerosp. Electron. Syst. Mag., vol. 29, no. 8, pp. 4-53, Aug. 2014.

[49] K. Levenberg, "A method for the solution of certain non-linear problems in least squares," Quarterly of Applied Mathematics, vol. 2, no. 2, pp. 164-168, Jul. 1944.

[50] D. W. Marquardt, “An algorithm for least-squares estimation of nonlinear parameters," Journal of the Society for Industrial and Applied Mathematics, vol. 11, no. 2, pp. 431-441, Jun. 1963.

[51] P. Zhang, L. Gan, S. Sun, and C. Ling, "Atomic norm denoising-based channel estimation for massive multiuser MIMO systems," in Proc. IEEE Int. Conf. Commun. (ICC), London, UK, Jun. 2015, pp. 4564-4569. 